\title{
Mechanisms for Lagged Atmospheric Response to ENSO SST Forcing*
}

\author{
Hui Su, J. David Neelin, and Joyce E. Meyerson \\ Department of Atmospheric Sciences, and Institute of Geophysics and Planetary Physics, University of California, Los Angeles, \\ Los Angeles, California
}

(Manuscript received 27 September 2004, in final form 1 March 2005)

\begin{abstract}
The mechanism and sensitivity of the lagged response of tropical tropospheric temperature to El NiñoSouthern Oscillation (ENSO) SST forcing are examined using the Quasi-Equilibrium Tropical Circulation Model (QTCM) coupled to a slab mixed layer ocean model, along with a simple analytical model. It is found that the lag and amplitude of tropospheric temperature response depend on mixed layer depth (MLD), ENSO SST forcing period, areal fraction of the mixed layer ocean, and the strength of Tropics to midlatitude transports. The phase lag is not a monotonic function of mixed layer depth. It maximizes at moderate MLD and, thus, is not very sensitive to MLD in the realistic range. The phase lag asymptotes to values determined by free-atmospheric time scales, between 1 and 2 months, for small or large values of MLD. The amplitude of the tropospheric temperature response decreases with increasing MLD. The phase lag and amplitude of tropospheric temperature both increase as a specified ENSO SST forcing period increases and they appear to be rather insensitive to the seasonal cycle of SST. On the other hand, the phase lag and amplitude of mixed layer ocean SST change monotonically with MLD and ENSO forcing period, with a deeper mixed layer producing longer lag and smaller amplitude of SST anomalies. Longer ENSO SST forcing periods correspond to longer lag and larger amplitude of mixed layer ocean SST anomalies. While the ENSO region convective heating (precipitation) anomalies are closely tied to SST anomalies, the tropical mean precipitation seems best viewed as a complex by-product of the response rather than as a driver. One useful parameter determining the lag of tropospheric temperature to ENSO SST is the freedecay time scale of the coupled system. This parameter combines the effects of surface flux exchanges, heat loss at the top of the atmosphere and from the Tropics to midlatitudes, and finite ocean heat capacity. It is indicative of the extent to which the lagged response of tropical tropospheric temperature to ENSO SST is a coupled phenomenon. Overall, the contribution of coupling to SST outside the ENSO region substantially increases the amplitude and lag of the tropospheric temperature response to ENSO.
\end{abstract}

\section{Introduction}

On interannual time scales, variations of tropical mean tropospheric temperature $\left(\left\langle\hat{T}^{\prime}\right\rangle\right)$ and related variables, such as geopotential height, tend to follow the changes of sea surface temperature (SST) in the equatorial eastern Pacific, with a lag of 1-2 seasons in maximum response (Newell and Weare 1976; Angell 1981; Pan and Oort 1983; Reid et al. 1989; Yulaeva and Wal-

\footnotetext{
* University of California, Los Angeles, Institute of Geophysics and Planetary Physics Contribution Number 6212.
}

Corresponding author address: J. David Neelin, Department of Atmospheric Sciences, University of California, Los Angeles, 405 Hilgard Avenue, Los Angeles, CA 90095-1565.

E-mail: neelin@atmos.ucla.edu lace 1994; Kumar and Hoerling 2003, hereafter KH03). On the other hand, a lagged relationship is also known for SST in other basins with respect to Pacific El NiñoSouthern Oscillation (ENSO) SST indices (Klein et al. 1999; Enfield and Mayer 1997; Tourre and White 1995; Latif and Barnett 1995). Previous research has suggested that this could occur via the atmospheric circulation teleconnected to the ENSO SST forcing, sometimes referred to as the "atmospheric bridge" (Alexander et al. 2002; Lau and Nath 1996; Klein et al. 1999). Because tropospheric temperature variability is important to the interbasin teleconnection associated with ENSO, this lagged relationship can have a complex effect on the precipitation response. For example, Chiang and Sobel (2002) studied the response of a singlecolumn model coupled to a slab mixed layer ocean to imposed tropospheric temperature variations. They 
found that the mixed layer SST responds to tropospheric temperature in a delayed fashion. For a deeper mixed layer depth, the SST lags the tropospheric temperature by a longer time, thus permitting greater surface flux variations and producing larger precipitation anomalies. Precipitation impacts via tropospheric temperature can also occur by the "upped ante mechanism" (Neelin et al. 2003), in which a warm troposphere increases the amount of low-level moisture required to maintain convection (the convective "ante"). This induces moisture gradients between convective and nonconvective regions, impacting precipitation at the margins of convective zones. Precipitation anomalies due to this mechanism have a different phase relationship to the coupled tropospheric temperature (and SST) response than those induced by surface flux mechanisms (Neelin and Su 2005).

Although the lagged thermal response of tropospheric temperature relative to ENSO SST has been noted in many studies using different observations and numerical model simulations, the mechanism for the lagged atmospheric response has yet to be understood. It is known that the tropical troposphere responds rapidly to an anomalous diabatic heating source, via convectively driven Kelvin waves (Heckley and Gill 1984; Jin and Hoskins 1995; Bantzer and Wallace 1996). The time scale associated with this is on the order of a week to 15 days. There must be other slower processes occurring to produce a lag of one to two seasons. $\mathrm{KH} 03$ examined the characteristics of the delayed atmospheric response to El Niño and proposed two different mechanisms for two features of the response. For the 1-3-month lag of the maximum tropospheric temperature with respect to maximum El Niño SST warming, they linked the evolution of tropospheric temperature with tropical mean rainfall anomalies and attributed the lag in diabatic heating to the interaction of ENSO SST anomalies with the seasonal cycle of SST; that is, the maximum diabatic heating occurs when total SST reaches a maximum. For the asymmetry of warm tropospheric temperature anomalies before and after the peak SST warming (lingering of warming after the peak SST anomalies), they suggested that the lagged response of the Indian and Atlantic SST plays an important role.

$\mathrm{Su}$ and Neelin (2003, hereafter SN03) pointed out that, on interannual time scales, the tropical mean rainfall anomalies $\left(\left\langle P^{\prime}\right\rangle\right)$ based on various satellite products show a poor relation (with large scatter) to tropical mean SST, even though tropospheric temperature has a clear linear relationship. Interpreting this using model results, SN03 argued that convection more directly constrains the relationship between tropospheric tempera- ture and SST, while the tropical mean precipitation anomalies are best thought of as a by-product of tropical atmosphere reaction to the SST forcing and the interaction with the midlatitudes.

In this study, we focus on the lag between the maximum tropical mean tropospheric temperature response and peak ENSO SST anomalies. We attempt to address the lagged tropospheric thermal response from an integrated coupled perspective, instead of a simple forced-damped process. We will provide a systematic examination of the sensitivity of the lagged response in an atmosphere model coupled with a mixed layer ocean and explore the mechanisms for the lagged response in an analytical framework. The hypothesis pursued here is as follows. Warmer than normal SST within the Pacific induces a warmer and moister boundary layer and enhances convection. Wave dynamics spreads the warming over the Tropics and creates widespread tropospheric temperature warming (Su et al. 2003, hereafter SNM03). The interaction with the ocean surface layer of finite heat capacity could slow part of the tropospheric warming. The modified surface flux affects SST, tropospheric temperature, and convection, while the top-of-atmosphere (TOA) radiation is important in the tropospheric budget.

The tool that we use is an intermediate climate model, known as the Quasi-Equilibrium Tropical Circulation Model (QTCM), coupled to a slab mixed layer ocean. The ENSO SST anomalies are prescribed and regarded as an external forcing to the system, similar to Alexander et al. (2002) and KH03. First, numerical experiments are examined to show the nature of the lagged response and sensitivity of lags to different model parameters. Second, a simple analytical framework is used to explore the fundamental time scales of the coupled system. Sobel and Gildor (2003) used a similar analytical model of the atmosphere plus a mixed layer ocean model. They examined the stability of the system to time-dependent oscillations and found that steady solutions of the model can become unstable to oscillations on intraseasonal to subannual time scales. We focus on the coupled solutions on interannual time scales. Analytical results are sought for the amplitude and phase of the response of the coupled system to prescribed ENSO SST forcing. The roles of individual physical processes are then analyzed and the mechanism for the lagged response is discussed.

This paper is organized as follows. Section 2 briefly describes the intermediate atmospheric model, the slab mixed layer ocean model, and experimental design. In sections 3 and 4, the observed and modeled lags of tropospheric temperature relative to the Niño-3.4 SST index are shown. Then the sensitivity of the lag to 
mixed layer ocean depth, period of SST forcing, and SST seasonal cycle is examined in section 5. Following the numerical results, section 6 presents the analytical model of the atmosphere-ocean coupled system and gives the amplitude and lag of tropospheric temperature as a function of mixed layer depth and period of SST forcing. The decay time scale of the coupled atmosphere-ocean solutions is given and used to indicate the lag in the coupled response. In section 7, the importance of tropical-midlatitude transports for the lag of tropospheric temperature is shown. Summary and discussions are given in section 8 .

\section{The QTCM and a slab mixed layer ocean model}

We use an intermediate complexity atmospheric circulation model, the QTCM, whose response to ENSO SST forcing has been validated in a number of previous studies (Zeng et al. 2000; Su et al. 2001; Su and Neelin 2002, hereafter SN02; SNM03). The QTCM is a primitive equation model that makes use of the properties of a quasi-equilibrium moist convective closure to simplify vertical structures of atmospheric profile (Neelin and Zeng 2000; Zeng et al. 2000). It includes nonlinear advection, Betts-Miller moist convective adjustment, cloud-radiative interaction, and a simple interactive land model.

The slab mixed layer ocean model used for this study assumes a fixed mixed layer depth (MLD). A range of values from 0.5 to $200 \mathrm{~m}$ for MLD are tested. The divergence of ocean transport is parameterized using the " $Q$ flux" correction scheme (Hansen et al. 1988, 1997) where time-averaged surface heat fluxes balance the ocean transport. The $Q$ flux is obtained from a control run with specified climatological SST. Climatological $Q$ flux is diagnosed from the seasonal surface fluxes and the time rate of change of SST in the control run and is used for all runs with a mixed layer ocean. In this study, we focus on the atmospheric circulation changes associated with ENSO SST forcing. Changes in ocean transport outside the ENSO region are not considered, although these may occur (Latif and Barnett 1995). The mixed layer ocean interacts with the atmosphere via daily averaged SST and surface fluxes.

A control run (ObsSST) is driven by global observed SST (Reynolds and Smith 1994) from 1950 to 2000. Ten simulations with slightly different initial conditions are conducted and ensemble means are constructed. Since the variability among the ten members of the ensemble is relatively small compared to ENSO-forced signals, some ensemble means consist of five members only. For runs ObsPac+ML, the QTCM is coupled with the mixed layer ocean except over the main ENSO anoma- lous SST region, where observed ENSO SST anomalies are used. On the contrary, run ObsPac uses climatological SST outside the ENSO SST region without an interactive ocean. The climatological SST refers to the seasonal cycle of SST based on observed monthly means from 1950 to 2000. The configuration of the specified ENSO SST region is based on the composite SST difference during December-February between seven El Niño events and six La Niña events over the period from 1950-2000. Its outline is approximately along the $1.0^{\circ} \mathrm{C}$ contour in the warm minus cold SST composite. The warm events include 1957/58, 1965/66, 1972/73, 1982/83, 1986/87, 1991/92, and 1997/98. The cold events include 1970/71, 1973/74, 1975/76, 1984/85, 1988/89, and 1998/99 (Alexander et al. 2002).

To test the sensitivity of the tropospheric temperature response to ENSO SST forcing, different mixed layer depths have been used, ranging from 0.5 to $200 \mathrm{~m}$. Besides using an observed SST anomaly as the specified forcing, an idealized sinusoidal SST anomaly is used in the SinPac+ML runs. In these runs, a different period of SST forcing and various mixed layer depths are used to provide a detailed picture of the sensitivity of the lag and amplitude of the tropospheric temperature response. We have also conducted an experiment in which the phase of the maximum SST anomaly is shifted from January to July to examine the role of a seasonal cycle. The sensitivity to an areal fraction of mixed layer ocean is tested by replacing part of the mixed layer ocean with climatological SST. Additional experiments are conducted in which anomalies of a particular physical process are suppressed to examine the role of such a process in affecting the lag and amplitude. For example, run ADVCLIM suppresses advection of temperature and moisture anomalies to estimate how importantly the energy and moisture transports between the Tropics and midlatitudes affect the tropospheric temperature response to ENSO SST forcing.

Our analysis focuses on the lead/lag regression of several variables to an ENSO SST index. We use the Niño-3.4 SST index (averaged for the area $5^{\circ} \mathrm{N}-5^{\circ} \mathrm{S}$, $170^{\circ}-120^{\circ} \mathrm{W}$ ) as the standard reference for the ENSO signal. The lag of a variable with respect to the Niño-3.4 SST is defined by the month when maximum regression occurs. This may be slightly different from that inferred by comparing two time series, the initiation of response, or other measures of phase relations. For observed tropospheric temperature, we use the National Centers for Environmental Prediction (NCEP)-National Center for Atmospheric Research (NCAR) reanalysis data (Kalnay et al. 1996). Tropospheric average refers to the mean between 850 and $200 \mathrm{hPa}$; tropical average refers to the mean between $25^{\circ} \mathrm{S}$ and $25^{\circ} \mathrm{N}$. 


\section{The observed and modeled tropospheric temperature lag}

Previous studies show that ENSO has a strong "phase locking" with a seasonal cycle (Rasmusson and Carpenter 1982), although considerable scatter exists for the ENSO onset and termination phases relative to the seasonal cycle (Neelin et al. 2000). The peak warming of El Niño usually occurs in the wintertime, around December and January. However, the maximum tropical tropospheric temperature warming does not occur until one to two seasons later. Figure 1 shows a lead/lag regression of zonally averaged tropospheric temperature anomalies onto the Niño-3.4 SST index for the NCEPNCAR reanalysis and a counterpart in the QTCM control run, ObsSST. The vertical axis labels from -12 to 12 , represent tropospheric temperature leading Niño3.4 SST 12 months to lagging Niño-3.4 SST 12 months.

For the NCEP-NCAR reanalysis (Fig. 1a), at lag -12 to -6 months (i.e., atmosphere leading Niño-3.4 SST 12-6 months), the tropospheric temperature appears to be negatively correlated with Niño-3.4 SST, likely due to the influence of the previous phase of the ENSO signal. Positive regression of tropical tropospheric temperature anomalies onto the Niño-3.4 SST occurs at all other lags, with a maximum at 3-4-month lag. The lead/lag regression of the modeled temperature onto Niño-3.4 SST (Fig. 1b) is similar to the observations. The maximum regression also occurs at 3 -month lag. However, the positive correlation of tropospheric temperature with Niño-3.4 SST begins in May of the year preceding the ENSO peak phase, sooner than the observed, possibly related to the weaker cold temperature anomalies simulated in the model. The regression coefficients are generally smaller for the ObsSST run than for the NCEP-NCAR reanalysis, and they are more confined in the equatorial region, especially at 4-10-month lags.

Figure 2 provides another view of the lagged response of tropospheric temperature to ENSO SST forcing. A time-latitude section of the composite zonally averaged tropospheric temperature anomalies for a $25-$ month period is shown, from January of the year preceding an El Niño warm event (month -12) to January of the year after the peak El Niño warming (month 12). The composite is based on four recent El Niño events since 1982, that is, the 1982-83, 1986-87, 1991-92, and 1997-98 El Niños. A composite sequence of 25 calendar months, centered on January, is contructed, averaging the four warm events. The January of the peak El Niño warm phase is labeled as month 0 . The composite Niño3.4 SST anomaly time series for the 25 months is displayed in Fig. 2a. Figure $2 \mathrm{~b}$ shows the composite for the

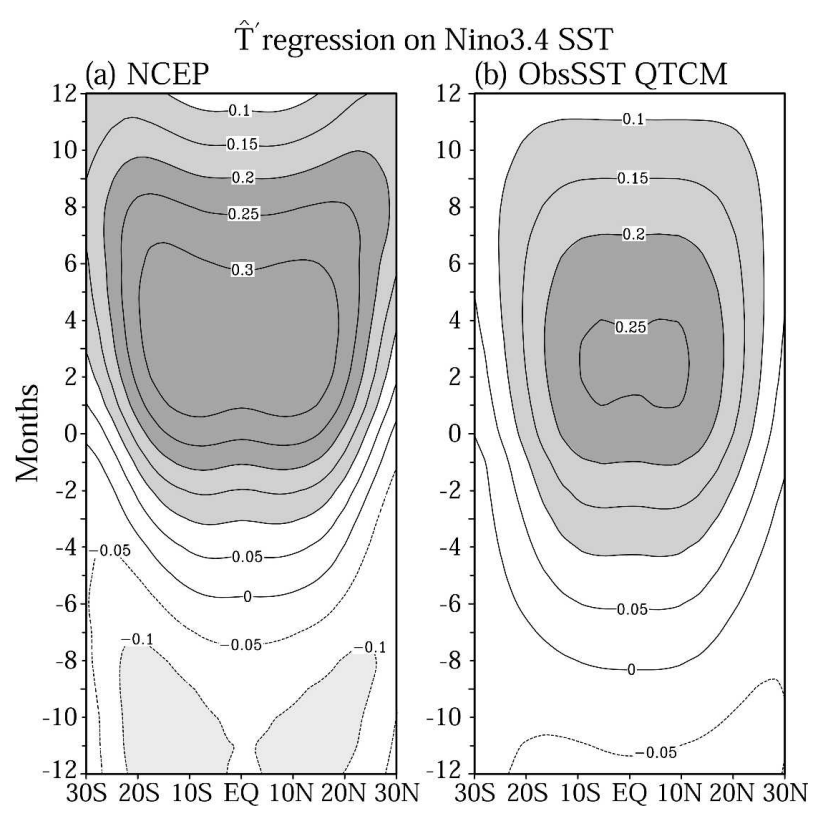

FIG. 1. Regression of zonally averaged tropospheric temperature onto the Niño-3.4 SST index for (a) the NCEP-NCAR reanalysis and (b) the QTCM run ObsSST. The lead/lag regressions are calculated relative to climatological means for the period from 1950 to 2000. Negative (positive) $y$-axis values indicate months for which the target variable leads (lags) the Niño-3.4 index. Negative contours are dashed. Regression values above (below) \pm 0.1 have medium (light) shading, with dark shading above 0.2 .

NCEP-NCAR reanalysis, and Fig. 2c shows the modeled composite for the ObsSST run. It can be seen that the warm phase of the composite Niño-3.4 SST anomaly starts in March (month -10) of year -1 and maximizes in January of year 0 . It reverses to cold anomalies from October (month 9) of year 0 and is approximately symmetric before and after the peak phase. However, the observed zonally averaged tropospheric temperature warm anomalies do not begin until May (month -8 ) of year -1 . The peak warming appears around March (month 2) of year 0 . The modeled tropospheric temperature response captures the lag in maximum warming with slightly weaker amplitude.

The horizontal distribution of simulated tropospheric temperature anomalies is also similar to the NCEPNCAR reanalysis. Figure 3 shows the composite tropospheric temperature anomalies at 2-month lag, that is, in March of year 0, for the NCEP-NCAR reanalysis (Fig. 3a) and the QTCM run ObsSST (Fig. 3b). Both modeled and observed composites exhibit a widespread warming across the Tropics. The spatial pattern is suggestive of an equatorial wave structure-an equatorially symmetric Rossby wave pattern in the eastern $\mathrm{Pa}$ cific and a Kelvin wave-like structure extending to the east. The modeled temperature response is somewhat weaker than observed. 
(a) SST Nino3.4

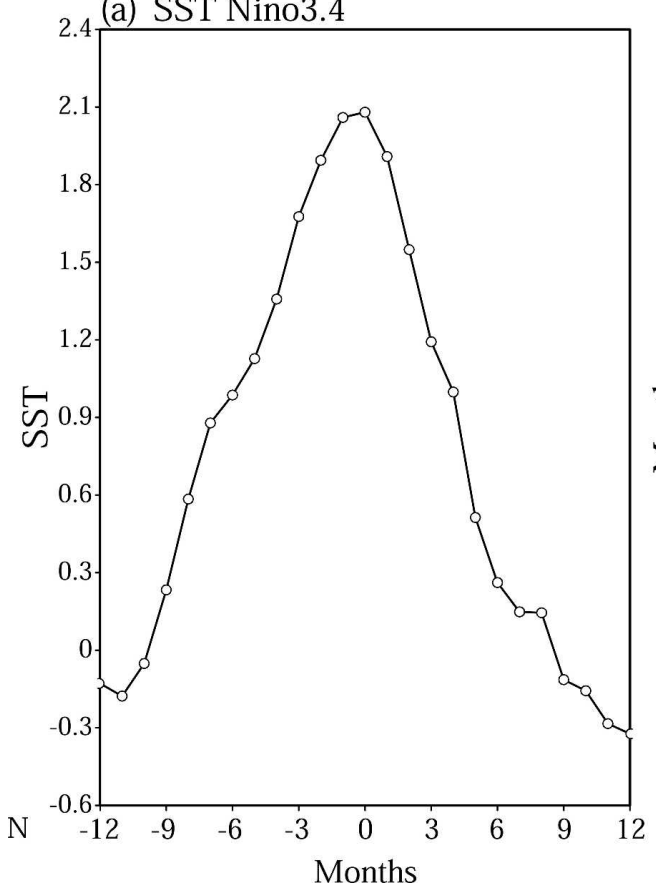

(b) $\hat{\mathrm{T}}^{\prime}$ NCEP

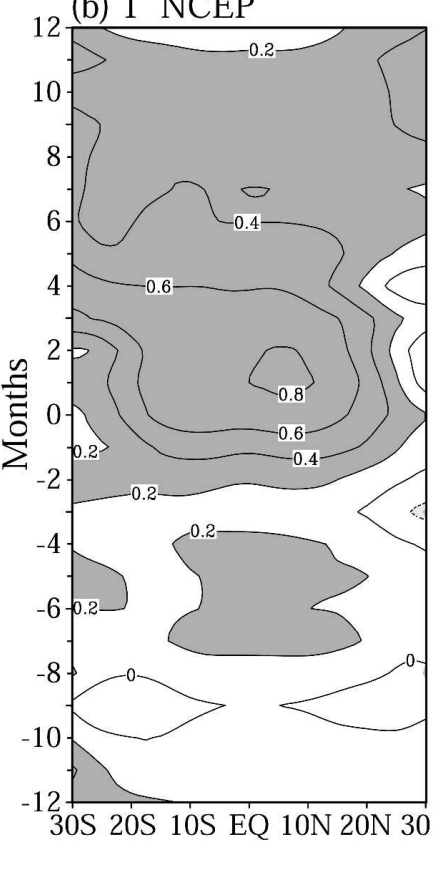

(c) $\hat{T}^{\prime}$ ObsSST QTCM

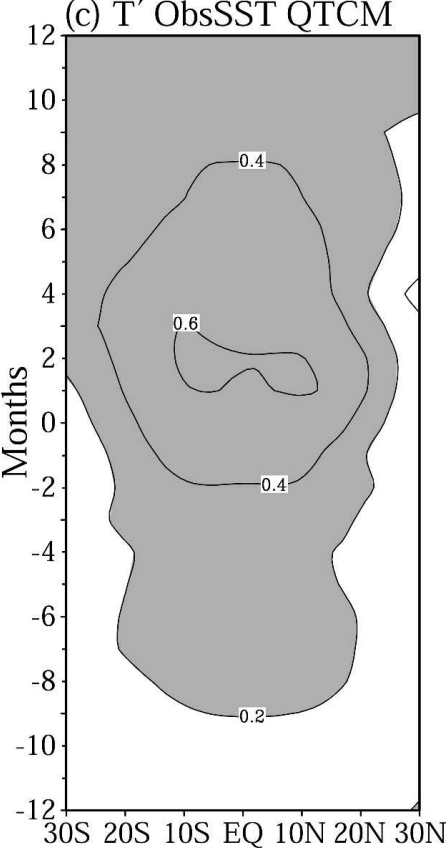

FIG. 2. Composite evolution of (a) anomalies of Niño-3.4 SST index, (b) zonally averaged tropospheric temperature anomalies for the NCEP-NCAR reanalysis, and (c) zonally averaged tropospheric temperature anomalies for the QTCM run ObsSST. The composite is based on the four El Niño events for the period of 1982-98. Month 0 refers to Jan of the El Niño warm years.

(a) $\hat{\mathrm{T}}^{\prime}$ Composite March Year 0 NCEP

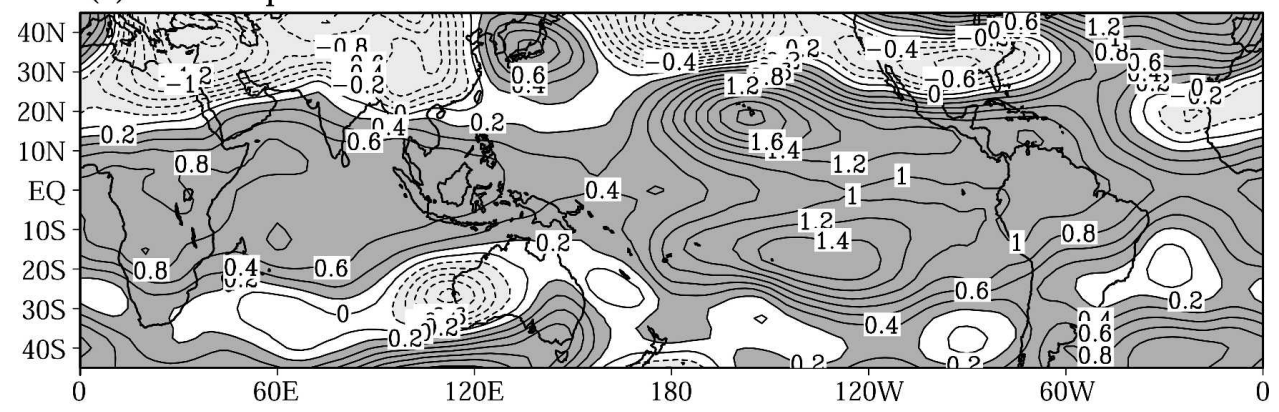

(b) $\hat{\mathrm{T}}^{\prime}$ Composite March Year 0 ObsSST QTCM

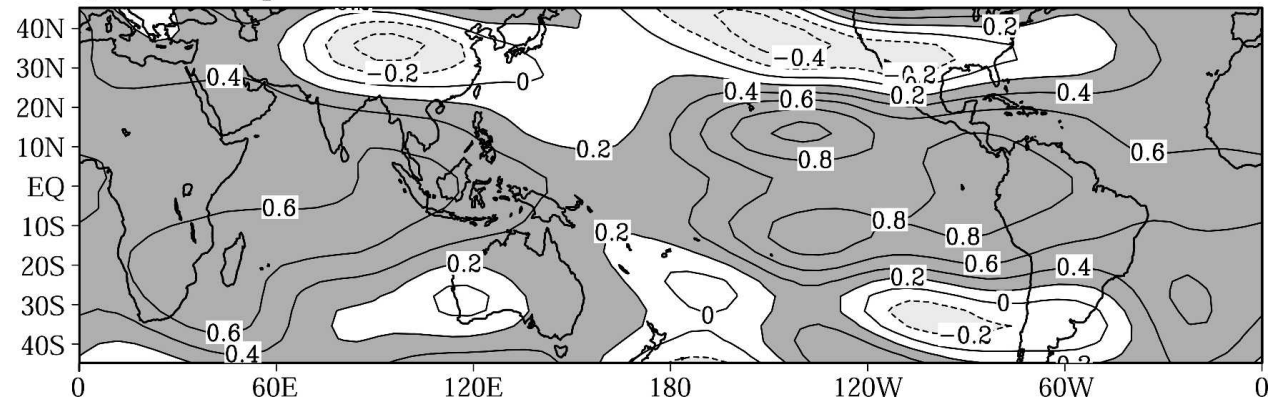

FIG. 3. Composite of zonally averaged tropospheric temperature anomalies in Mar of the El Niño years (month 2 in Fig. 2), close to the peak tropospheric temperature warming for (a) the NCEP-NCAR reanalysis and (b) the QTCM run ObsSST. Composite is based on the four El Niño events from 1982 to 1998 . Positive anomalies greater than $0.2^{\circ} \mathrm{C}$ are shaded. 
(a) SST 5-month lag regression to Nino3.4 OBS

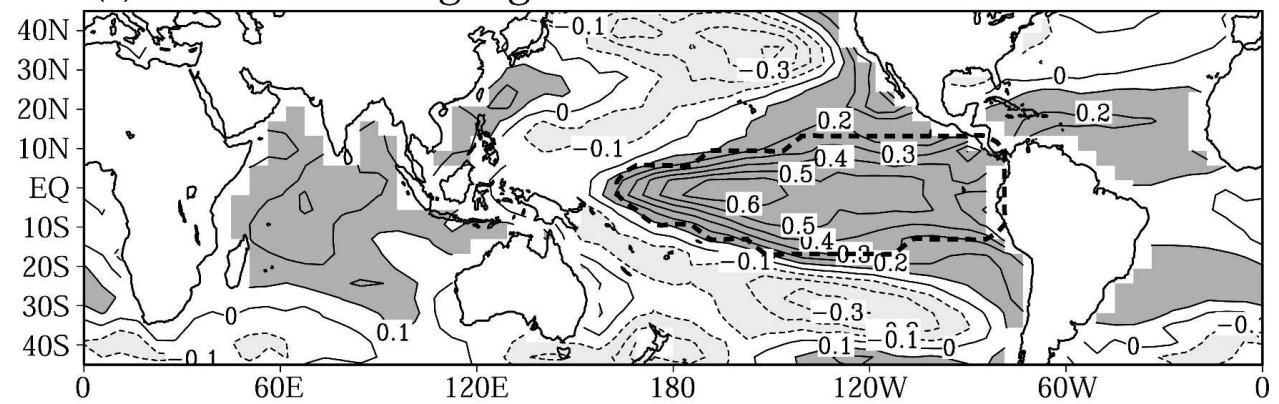

(b) ObsPac $+\mathrm{ML}(25 \mathrm{~m})$

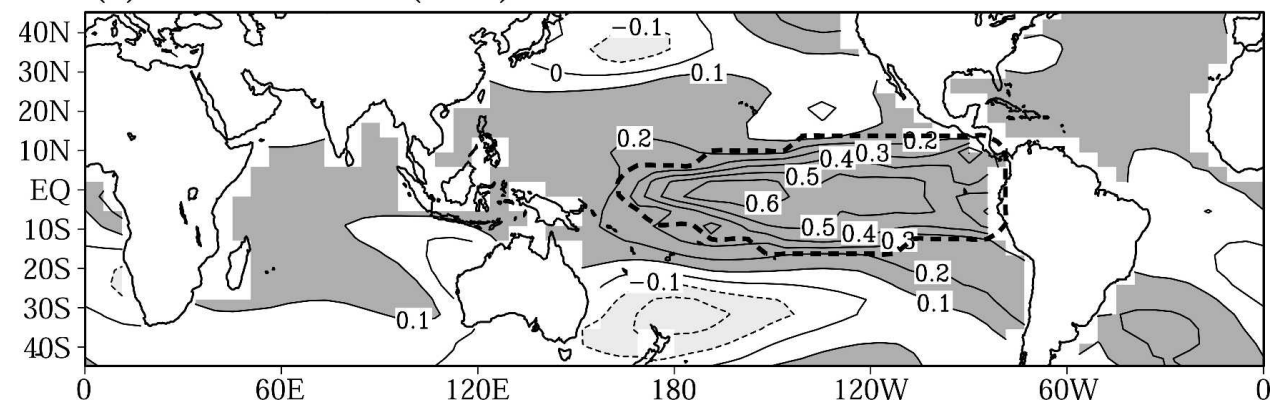

FIG. 4. Regression of SST onto the Niño-3.4 SST index at 5-month lag for (a) observed SST and (b) simulated SST in the run ObsPac + ML, with a mixed layer depth of $25 \mathrm{~m}$. Dark (light) shading above (below) \pm 0.1 . Contour interval is $0.1^{\circ} \mathrm{C}$. The dark dashed outline indicates the region of specified observed SST.

\section{Lagged response in the coupled atmosphere-mixed layer ocean model}

A set of experiments, referred to as ObsPac+ML, are conducted to examine the tropospheric temperature lag in the coupled context of atmosphere and mixed layer ocean. The observed ENSO SST forcing is prescribed within the composite SST anomaly region, and the mixed layer ocean is used elsewhere.

The simulated mixed layer ocean SST lags the Niño3.4 by a season or two. Figure 4 shows the regression of global SST anomalies onto the Niño-3.4 SST index at a 5-month lag for observations and for the QTCM run ObsPac + ML with a mixed layer depth of $25 \mathrm{~m}$. The dark outline indicates the region of specified observed SST. Note that the regression inside the ObsPac box is identical for the observations and the model by design. Cold SST anomalies in the North and South Pacific are simulated, although their locations are restricted to midlatitudes and maxima occur westward of the observed. The cold SST anomalies in the tropical western Pacific are not simulated, possibly owing to the lack of ocean dynamics there. The Indian Ocean and tropical North Atlantic Ocean SSTs both positively correlate to ENSO SST at this lag, with the modeled amplitude about half of the observed. It has been suggested that ocean dynamics and/or local air-sea interaction may be important for the Indian Ocean SST variability (Webster et al. 1999; Yu and Rienecker 2000; Yu et al. 2002). Thus, the atmospheric model coupled with a mixed layer ocean may not be able to fully capture the SST response.

Figure 5 shows the simulated tropospheric temperature anomaly composite in March of El Niño years from the same ObsPac $+\mathrm{ML}$ run. The temperature pattern resembles that in Fig. 3, with a larger positive anomaly area in the western Pacific than in Fig. 3, possibly due to the missing simulated cold SST anomalies there. Nonetheless, the widespread tropospheric temperature warming is reproduced with reasonable strength.

For the ObsPac + ML run with a mixed layer depth of $25 \mathrm{~m}$, the maximum regression of tropical averaged tropospheric temperature anomalies onto the Niño-3.4 SST index occurs at a 2-month lag, 1 month shorter than in the ObsSST run. For comparison, we conduct the ObsPac run, where climatological SST replaces the mixed layer SST in areas outside of the specified ENSO SST. Without an interactive ocean, the lag of tropical tropospheric temperature is reduced to 1 month. Figure 6 shows the resulting lead/lag regression of tropical averaged tropospheric temperature $\left(25^{\circ} \mathrm{S}-25^{\circ} \mathrm{N}\right)$ with re- 


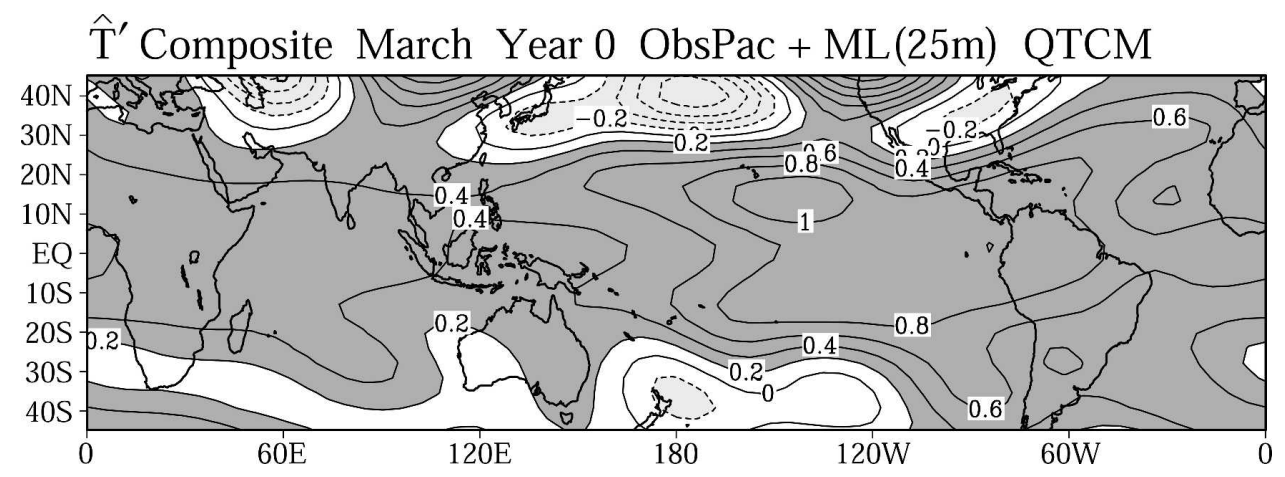

FIG. 5. As in Fig. 3, but for the QTCM run ObsPac+ML (MLD = $25 \mathrm{~m})$.

spect to Niño-3.4 SST for the ObsSST, ObsPac+ML $(25 \mathrm{~m})$, and ObsPac runs as well as that for the NCEPNCAR reanalysis. Clearly, without an active mixed layer ocean, the lag of tropospheric temperature is relatively short and the amplitude of temperature anomalies is also small compared to model runs with a mixed layer ocean or observed SST. The results are consistent

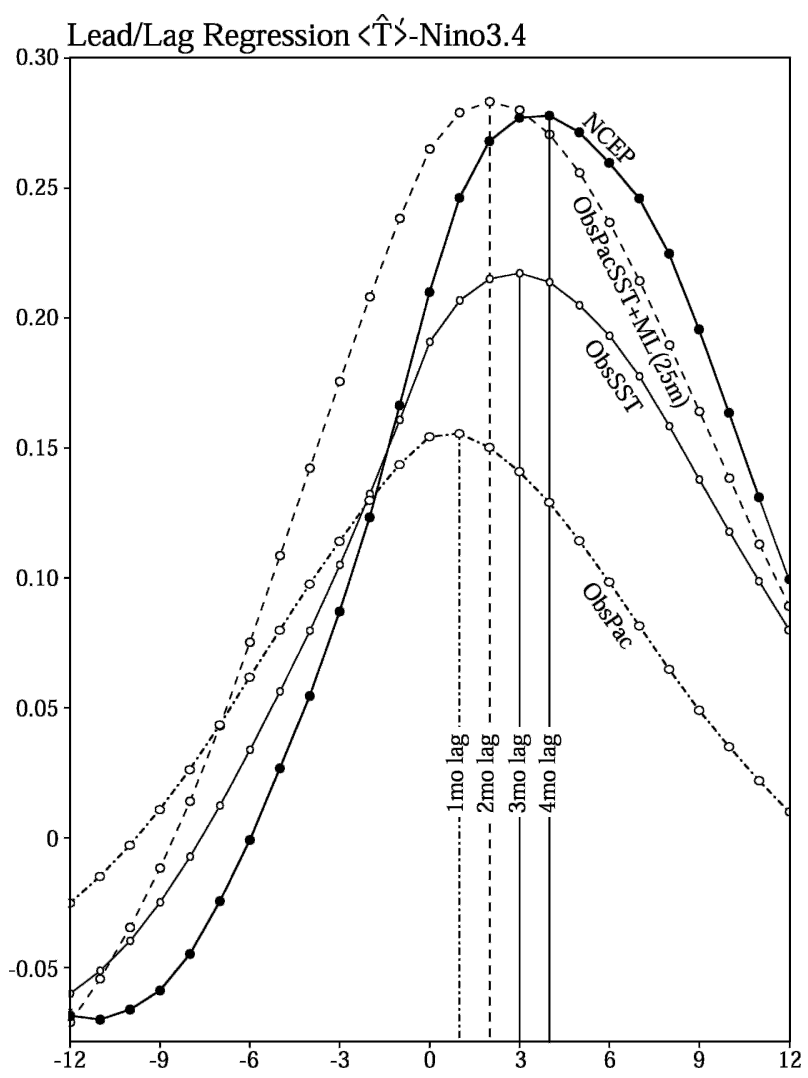

FIG. 6. Lead/lag regression coefficients of tropical tropospheric temperature anomalies onto the Niño-3.4 SST index for the NCEP-NCAR reanalysis, the QTCM run ObsSST, ObsPac $+\mathrm{ML}(\mathrm{MLD}=25 \mathrm{~m})$, and run ObsPac. Corresponding lags for maximum regression values are marked. with KH03 (their Fig. 10). They found that the lag in the atmospheric geopotential field exists, even without using a mixed layer ocean, and concluded that the lag is not a coupled phenomenon (although their run with a mixed layer ocean produced a longer lag than their climatological SST run). The ObsSST run has the closest lag to the NCEP-NCAR reanalysis data among three model experiments, although its amplitude of tropospheric temperature anomalies is about $20 \%$ lower than observed. The ObsPac + ML run with a $25-\mathrm{m}$ mixed layer depth produces a similar magnitude of regression of $\left\langle\hat{T}^{\prime}\right\rangle$ to Niño-3.4 SST, but its lag is 2 months shorter than observed. The larger tropospheric temperature response in the ObsPac+ML run than that in the ObsSST run may be related to the missing cold SST anomalies simulated in the model. An experiment with the observed SST specified in the entire tropical Pacific and mixed layer ocean outside confirms that inclusion of cold SST anomalies in the western Pacific would reduce the tropical mean tropospheric temperature anomalies by $20 \%-30 \%$. We note in Fig. 6 that the regression coefficients are not symmetric about the peak, a phenomenon noted in KH03. Experiments in section $5 \mathrm{~b}$ make clear that this asymmetry is a property of the observed ENSO region SST, and not of the troposphere plus mixed layer ocean response.

In the above experiments, the coupled atmosphereocean system responds to a specified interannual SST forcing in a delayed fashion. The finite heat capacity of the mixed layer ocean clearly plays a role, but sensitivity to mixed layer depth and other model parameters is of interest.

\section{Sensitivity of the lagged response}

For the sensitivity experiments, we use two types of interannual SST forcing. First, the observed SST time series within the composite ENSO region (as shown in Fig. 4) are used in the ObsPac + ML runs. The mixed 
Lead/Lag Regression $\langle\hat{T}\rangle^{\prime}-$ Nino3.4

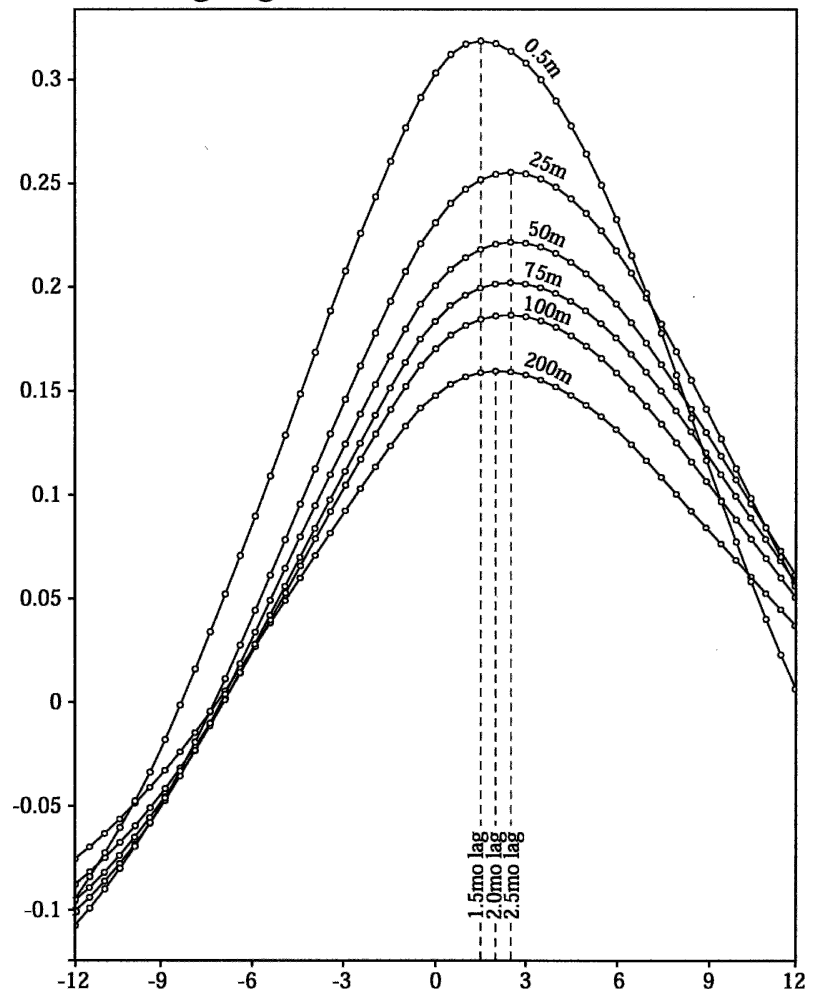

FIG. 7. Lead/lag regression coefficients of tropical tropospheric temperature anomalies onto the Niño-3.4 SST index for the QTCM runs ObsPac + ML at different mixed layer depths ranging from 200 to $0.5 \mathrm{~m}$. Corresponding mixed layer depths are shown above the curves, and lags for maximum regression values are marked.

layer depth is varied from 0.5 to $200 \mathrm{~m}$. Another set of experiments, referred to as $\mathrm{SinPac}+\mathrm{ML}$, use an idealized sinusoidal SST forcing with varying periods at 2, 3, and $4 \mathrm{yr}$. This aims to identify how the periods of SST forcing impact the lag and amplitude of the tropospheric temperature and SST response.

\section{a. Sensitivity to mixed layer depth}

Figure 7 shows the lead/lag regression of tropical averaged tropospheric temperature onto the Niño-3.4 SST index for the ObsPac+ML runs with a MLD of 0.5 , $25,50,75,100$, and $200 \mathrm{~m}$. The regression is based on model results from 1982 to 2000, which is different from the analysis period used for Fig. 6, which is from 1950 to 2000. The model output resolution is biweekly in Fig. 7, finer than that in Fig. 6 (monthly). It is shown that the lags of tropospheric temperature at a MLD of 25, 50, 75 , and $100 \mathrm{~m}$ are all 2.5 months, compared to a lag of 1.5 months for a MLD of $0.5 \mathrm{~m}$ and a lag of 2 months for a MLD of $200 \mathrm{~m}$. Differences in lags less than 2 weeks cannot be detected based on biweekly model outputs. The smaller lag for the run with a MLD of $0.5 \mathrm{~m}$ is expected owing to the smaller heat capacity of the ocean. The amplitude of the tropospheric temperature response is higher for smaller MLD. On the other hand, the shorter lag at an MLD of $200 \mathrm{~m}$ than those for shallower MLDs seems counterintuitive. However, we note that the thermal response is smaller when MLD is $200 \mathrm{~m}$ than other runs; thus, the impact of the ocean onto the troposphere would be smaller than the other runs as well. Considering an extreme case in which the ocean heat capacity is infinitely large, this mixed layer ocean is equivalent to a fixed-SST lower boundary. Thus, the coupled system would tend to have a response time scale solely determined by the atmosphere. It would be shorter than that of the coupled system with moderate MLD. Observed MLDs of the tropical ocean range from $10 \mathrm{~m}$ to $100 \mathrm{~m}$ (Watterson 2002). The 200-m hypothetical MLD thus suggests an extreme case behavior for the response of the coupled atmosphereocean system to interannual SST forcing. Although the lags for tropospheric temperature are not a monotonic function of the mixed layer depth, the amplitude of the temperature response appears to increase monotonically as MLD decreases. The amplitude variations with MLD changes are implicit in prior work with a simple atmosphere-mixed layer ocean system (e.g., Barsugli and Battisti 1998). Sobel and Gildor (2003) found that the response of their simple atmosphere-mixed layer ocean model to intraseasonal disturbance has a nonmonotonic dependence on mixed layer depth, with maximum response occurring at a mixed layer depth of $10-20 \mathrm{~m}$.

The measure of the lag used above refers to the phase delay of the peak response of the tropical tropospheric temperature anomalies with respect to Niño-3.4 SST. The variations of the so-defined lag for MLD between 0.5 and $200 \mathrm{~m}$ are within a month, even though the lead/lag regression curves in Fig. 7 appear to be significantly different for MLDs at 0.5 and $200 \mathrm{~m}$. Choosing other definitions for lag may result in different numerical values of the lags. For example, using the timing of the half-amplitude $\left\langle\hat{T}^{\prime}\right\rangle$ response to define lag, the difference between a MLD at 0.5 and $25 \mathrm{~m}$ would be about 1.5 months. Considering the asymmetry of the lead/lag regression curve may also provide another measure of lag. For simplicity, we will continue to use the lag of the peak response as our standard measure.

For the mixed layer ocean SST, the lag relative to Niño-3.4 is longer than the counterpart for tropospheric temperature. Figure 8 shows the lead/lag regression of the simulated mixed layer SST outside the ENSO region. The observed tropical mean SST outside the 


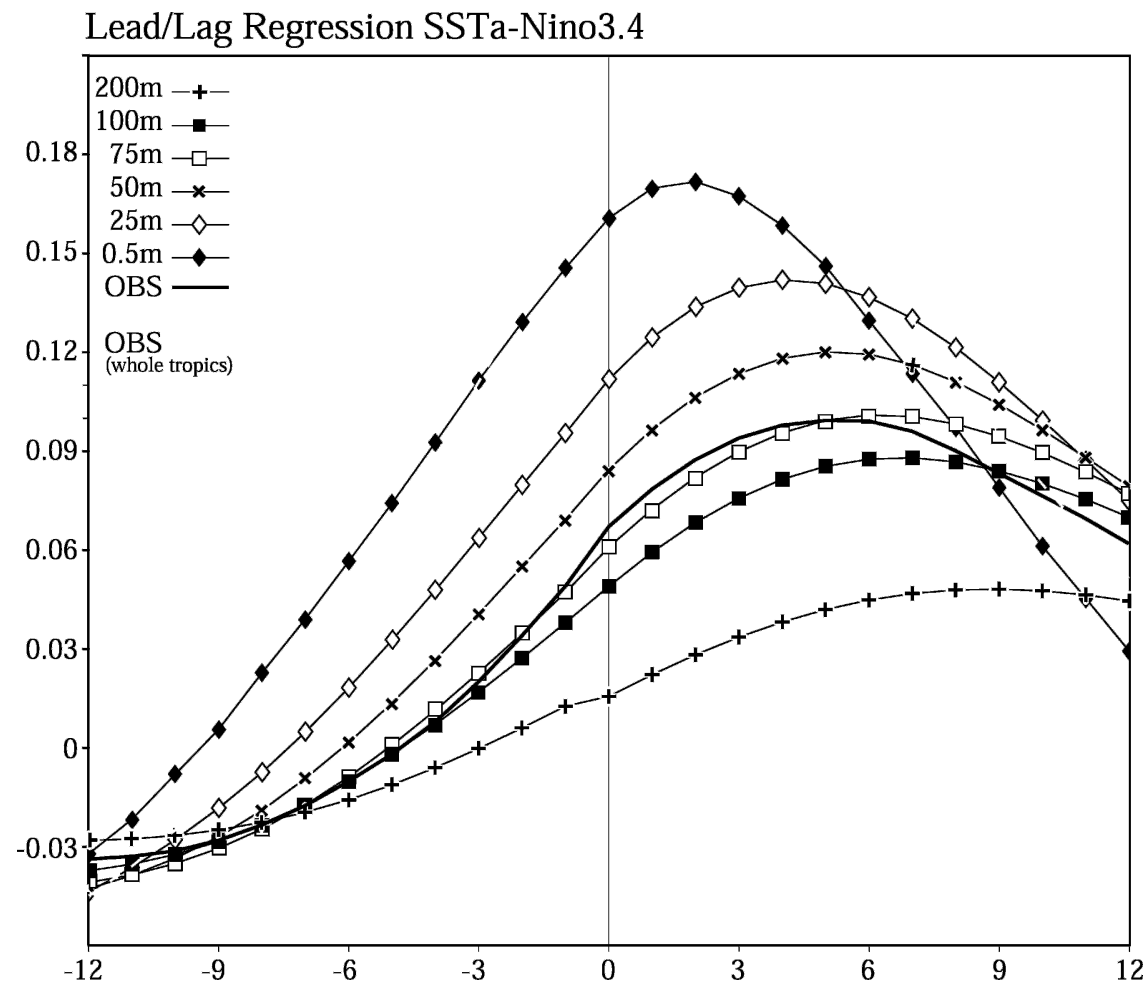

FIG. 8. Lead/lag regression coefficients of averaged mixed layer SST onto the Niño-3.4 SST index for the QTCM runs ObsPac+ML. The dark solid line is for observed ocean SST outside the specified ENSO SST region and the light gray dashed line refers to SST anomalies averaged over the whole Tropics.

ENSO region and over the whole Tropics is also plotted. The observed tropical mean SST has a relatively small lag to Niño-3.4 SST because of the dominance of El Niño SST in the average; however, the observed SST outside the ENSO region shows a large lag of about 5 months. The modeled mixed layer ocean SSTs lag the Niño-3.4 SST 2-10 months, with increasing lag for increasing MLD. The amplitude of the SST response, on the other hand, decreases as MLD increases. For a MLD of $25 \mathrm{~m}$, the lag is 4 months; while for an MLD of $50 \mathrm{~m}$, the lag is 5 months, comparable to that observed. The amplitude of SST anomalies is larger than that observed in the ObsPac+ML ( $25 \mathrm{~m}$ or $50 \mathrm{~m}$ ) runs, possibly due to the lack of simulated cold SST anomalies in the western Pacific (see Fig. 4).

Because convection is an important player in communicating boundary layer forcing to troposheric temperature warming, it is worth noting how precipitation varies in the coupled system response. Figure 9 shows the lead/lag regression of the tropical averaged precipitation for the ENSO region, outside the ENSO region, and for the whole Tropics. Both precipitation anomalies inside and outside the ENSO region more or less follow the Niño-3.4 SST variations, with little lag behavior. The large cancellation of the positive precipitation anomalies within the ENSO region and negative anomalies outside gives a rather small tropical mean precipitation anomaly. The lag in tropical mean precipitation anomaly tends to be between the lag of tropospheric temperature and that of mixed layer ocean SST. For realistic mixed layer depths the $\left\langle\hat{T}^{\prime}\right\rangle$ and $\left\langle P^{\prime}\right\rangle$ lags are fairly close, so this might be hard to distinguish in observations. Since the tropical mean rainfall has a different lag behavior than tropospheric temperature, one can argue against viewing it as a driving force for the tropospheric temperature lag. The complex relation of tropical mean precipitation lag to other parameters will be examined further in the analytical model (section $6 \mathrm{~d}$ ).

The amplitude of the precipitation response varies with mixed layer depth. For deeper mixed layer depth, the amplitude of precipitation anomalies (both inside and outside the ENSO forcing) is larger. This is consistent with Chiang and Sobel (2002). The physical processes responsible for this sensitivity are of interest and will be examined further in future work. 


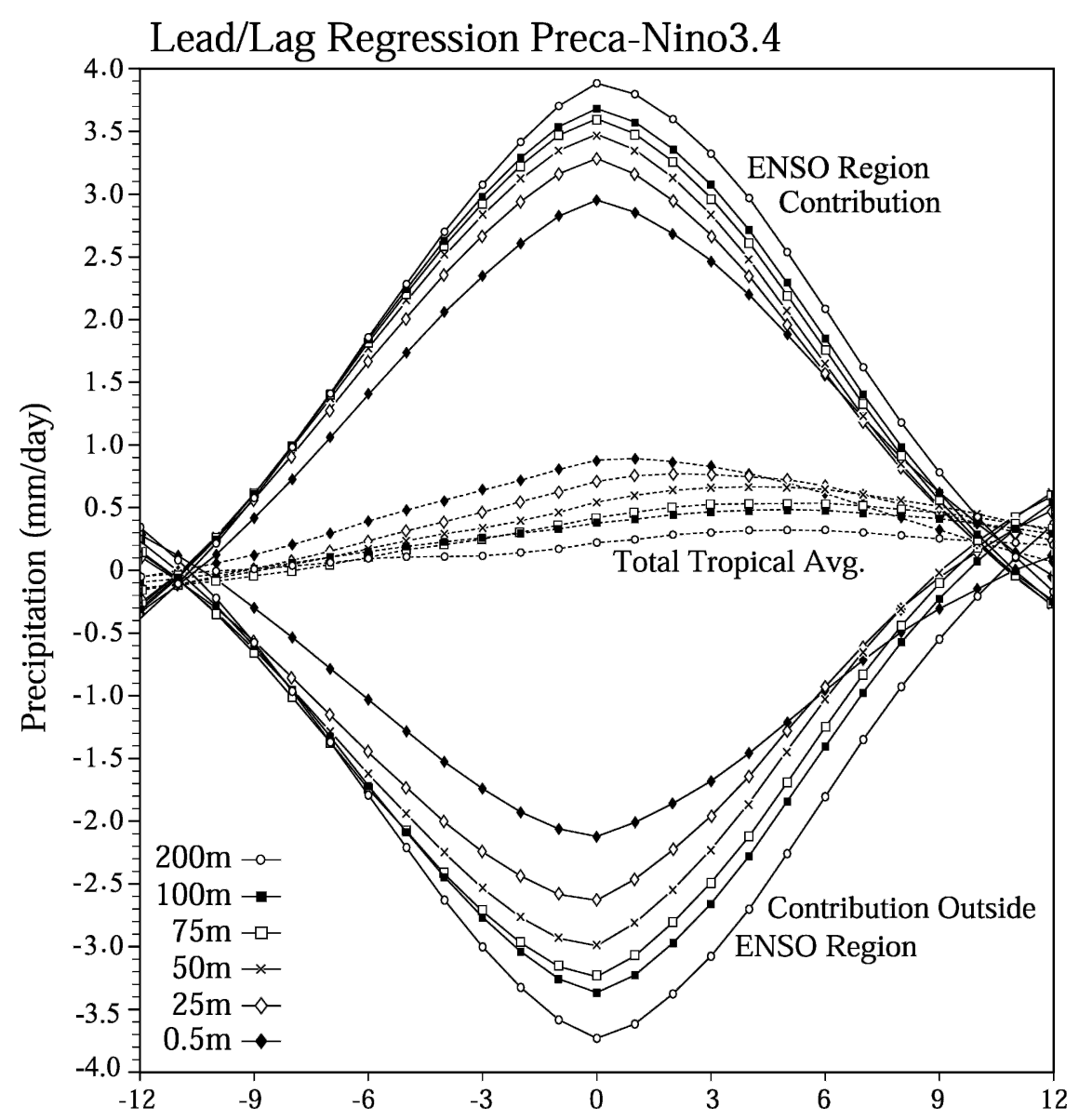

FIG. 9. Similar to Fig. 7 but for averaged precipitation anomalies outside and inside the specified ENSO SST forcing region (expressed as contributions to the tropical average) as well as tropical average precipitation $\left(25^{\circ} \mathrm{S}-25^{\circ} \mathrm{N}\right)$.

\section{b. Sensitivity to the period of ENSO SST forcing}

In the SinPac + ML runs, a sinusoidal SST anomaly is specified over the composite ENSO SST region. It is of the form

$$
T_{s}=T_{s 0} \sin \left(2 \pi t / \tau+\phi_{o}\right),
$$

where $\tau$ is the period of the SST anomaly forcing, with values of 2,3 , and $4 \mathrm{yr}$. The initial phase $\phi_{o}$ is chosen to give a peak warming in January. The spatial form and amplitude of the forcing $T_{s 0}$ is based on a composite of ENSO SST anomalies, that is,

$$
\frac{1}{2}\left(T_{s}^{\text {warm }}-T_{s}^{\text {cold }}\right),
$$

where $T_{s}$ is January SST averaged for warm/cold years.

Figure 10a shows time evolution of the specified SST forcing for a period of $2 \mathrm{yr}$ in the run with an MLD of $50 \mathrm{~m}$ and the corresponding time series for the tropical averaged tropospheric temperature, mixed layer ocean
SST, and precipitation inside and outside of the prescribed SST region. The simulated tropospheric temperature anomalies exhibit a semisinusoidal character, with a slight departure from a strict sinusoidal curve. It is clear that tropospheric temperature anomalies and mixed layer SST both lag the specified SST forcing, with the ocean responding in a much slower fashion. The lead/lag regression of these variables relative to the SST forcing is shown in Fig. 10b. The tropospheric temperature exhibits a lag of 2 months and the SST has a lag of 4 months. Note that the temperature response before and after the peak $\left\langle\hat{T}^{\prime}\right\rangle$ warming is rather symmetric, which is different than when observed ENSO SST forcing is used (Fig. 7). KH03 pointed out a distinction between the lag of tropospheric temperature as measured by the peak regression coefficient and a longer "lingering" of warm tropospheric temperature after the peak warming. In the context of these model simulations we can make precise statements relevant to this by defining asymmetry of the tropospheric tem- 
(a) 2 yr. SinPac+ML(50m) Time Evolution

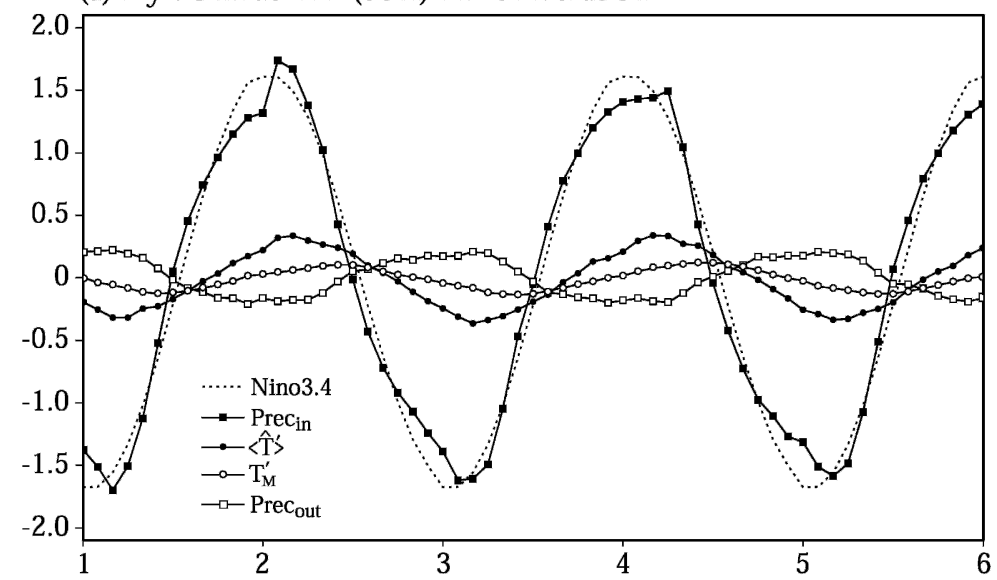

(b) Lead/Lag Regression 50m MixLayer (2yr. SinPac+ML(50m))

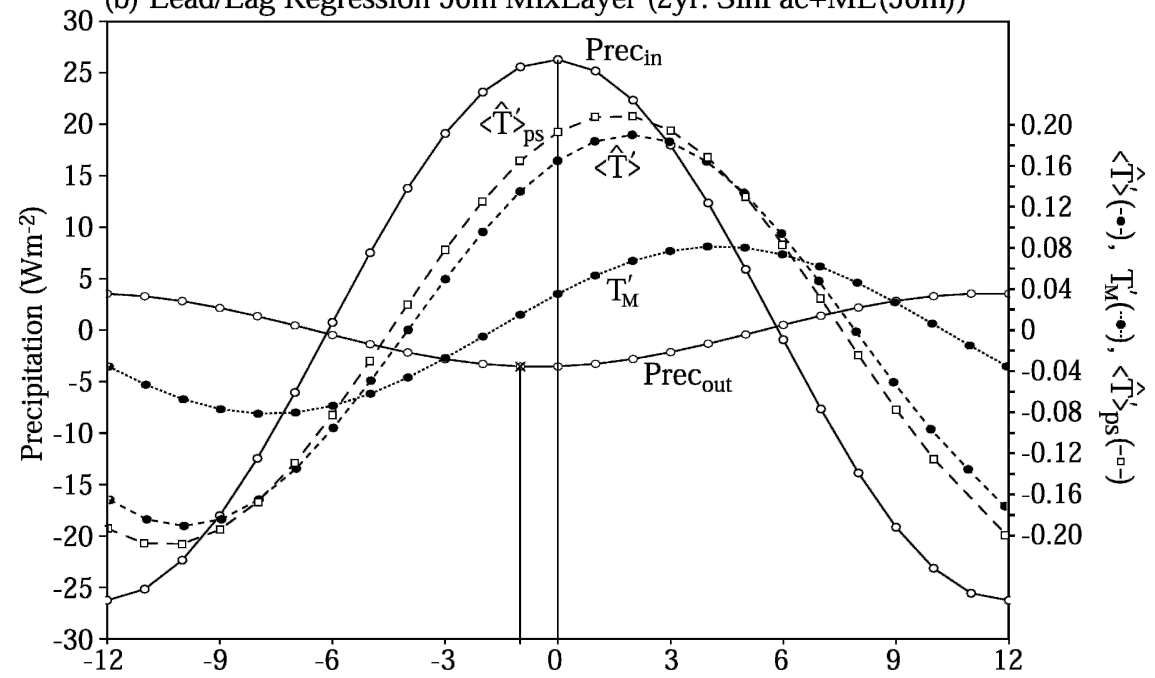

FIG. 10. (a) Time series of model simulated anomalies of area-averaged mixed layer SST $\left(T_{M}^{\prime} ;\right.$; in $\left.{ }^{\circ} \mathrm{C}\right)$, tropical mean tropospheric temperature $\left(\langle\hat{T}\rangle^{\prime} ;\right.$ in $\left.{ }^{\circ} \mathrm{C}\right)$, precipitation (in mm day ${ }^{-1}$ ) averaged inside $\left(\mathrm{Prec}_{\mathrm{in}}\right)$ and outside $\left(\mathrm{Prec}_{\mathrm{out}}\right)$ the SST forcing mask regions from a SinPac + ML run with a MLD of $50 \mathrm{~m}$ and SST forcing period of $2 \mathrm{yr}$. The specified SST forcing is indicated by the time series of the Niño-3.4 SST index. (b) Lead-lag regression coefficients of the variables in (a) onto the Niño-3.4 SST index. The curve marked by $\langle\hat{T}\rangle_{p s}^{\prime}$ refers to the simulated tropospheric temperature lead-lag regression when the peak phase of SST forcing is shifted by 6 months.

perature response relative to its own maximum. In Fig. $10 \mathrm{~b}$, the onset and decay of the warming in $\left\langle\hat{T}^{\prime}\right\rangle$ regression coefficients are quite symmetric about the maximum and the phase shift measured by the peak is characteristic of the phase shift of the whole, fairly sinusoidal, pattern. In Fig. 7, this is not the case. For instance, for the 50-m case, the zero regression crossing occurs approximately 9.5 months before the $\left\langle\hat{T}^{\prime}\right\rangle$ maximum, whereas 9.5 months after it the regression coefficient is about 0.052 , roughly a quarter of the peak value. Since this does not occur in the SinPac+ML experiment, it must be largely a property of the ENSO region SST forcing in the ObsPac experiment.
The precipitation anomalies within the ENSO forcing region in Fig. 10b are more closely concurrent with the evolution of SST forcing, with maximum regression occurring at 0 lag. The averaged precipitation anomaly outside the SST mask (Fig. 10b) has a smaller amplitude and the minimum occurs 0 to 1 month prior to the Niño-3.4 SST maximum (the regression coefficients are barely distinguishable for the 1-month lead and 0 lag). This again confirms that diabatic convective heating is not a driving force to the tropospheric temperature changes, but rather a result of moist convective adjustment to lower boundary forcing. The slight lead of the precipitation minimum may be understood as a com- 
promise between the loss of surface flux into the ocean (which occurs where $\partial_{t} T_{M}^{\prime}$ is maximum in Fig. 10b) and other effects, such as the "upped-ante mechanism" (Neelin et al. 2003; Neelin and Su 2005), which causes the largest precipitation anomalies roughly when tropospheric temperature maximizes.

Figures 11 and 12 summarize the modeled phase lag and amplitude of the tropospheric temperature and mixed layer ocean SST responses as a function of mixed layer depth and the period of SST forcing for all $\mathrm{SinPac}+\mathrm{ML}$ runs. The lags are quantized at 0.5 month by the output interval. Overall, the phase lag of tropospheric temperature is not a monotonic function of MLD. The amplitude of tropospheric temperature, on the other hand, changes rather monotonically with MLD, with a deeper mixed layer ocean yielding a weaker response. Both phase lag and amplitude increase as the period of SST forcing increases. For mixed layer ocean SST (Fig. 12), the phase lag increases as the MLD and SST period increase. The am-
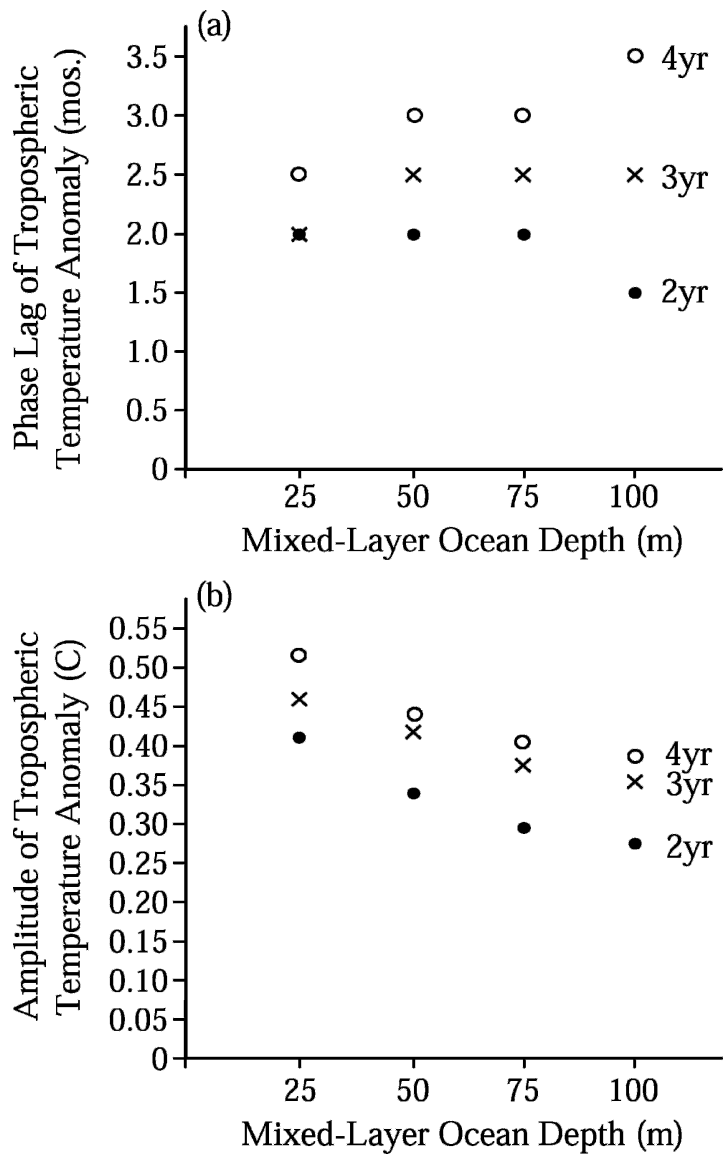

FIG. 11. (a) Phase lag (months) and (b) amplitude $\left({ }^{\circ} \mathrm{C}\right)$ of tropical average tropospheric temperature anomalies as functions of mixed layer depth and period of idealized SST forcing for all QTCM SinPac + ML runs.
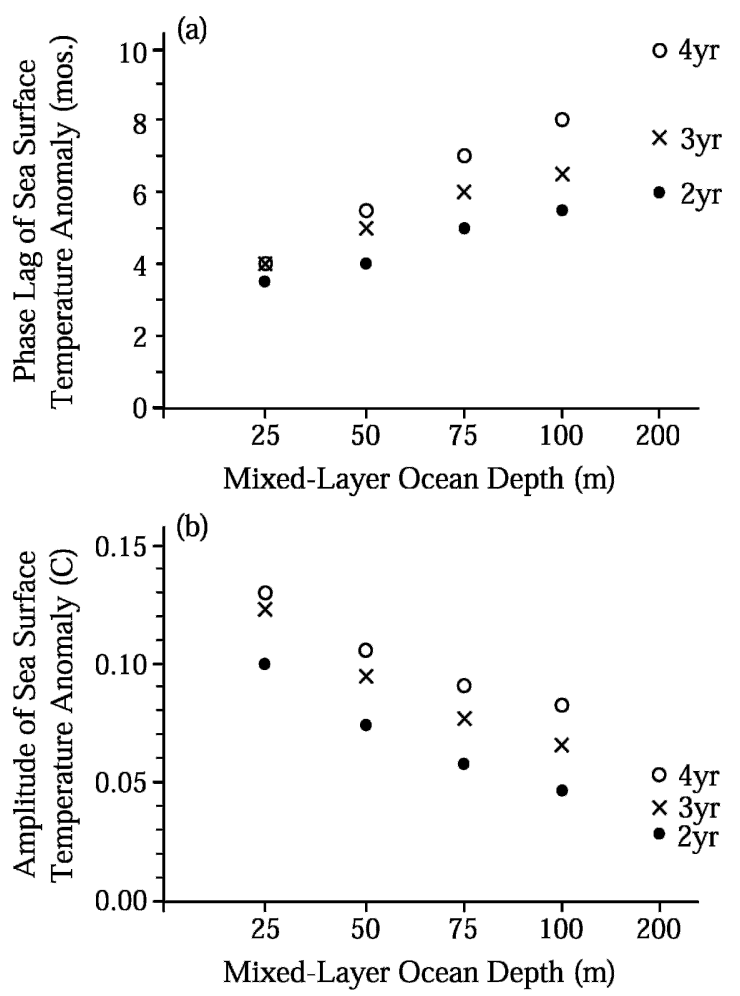

FIG. 12. As in Fig. 11, except for model-simulated mixed layer SST.

plitude of mixed layer SST anomaly decreases as MLD increases and increases when the SST forcing has a longer period. Note that, if phase lag scaled by the SST forcing period is plotted as a function of MLD scaled by period (see appendix B), the curves do not overlap. This indicates that time scales other than that given by mixed layer heat capacity, for example, atmospheric wave dynamics, must come into play.

\section{c. Sensitivity to SST seasonal cycle}

KH03 mentioned that lag of the tropospheric thermal response with respect to ENSO SST may be related to the interaction of SST anomalies with the seasonal cycle of SST. To test this, we conduct an experiment in which the initial phase $\phi_{o}$, as in (5.1), is chosen to give a peak SST warming in July, instead of January. The resulting tropospheric temperature lead/lag regression with the specified forcing is shown in Fig. 10b (indicated by $\left.\left\langle\hat{T}^{\prime}\right\rangle_{p s}^{\prime}\right)$. The phase lag is very similar to the standard run with peak SST warming in January, suggesting that the seasonal cycle is not crucial to the lagged response of tropospheric temperature. An experiment with peak SST warming in July and with climatological SST prescribed in regions outside the ENSO SST forcing yields a lag similar to the run with peak SST warming in Janu- 
ary. It again confirms that the interaction of SST anomalies and the SST seasonal cycle is not central to the lag of tropospheric temperature response.

\section{d. Sensitivity to the areal fraction of mixed layer ocean}

It is natural to think that the heat capacity of the mixed layer ocean is a control factor for the lag of the coupled system response. Thus, there should exist sensitivity of lags to the area fraction of the mixed layer ocean in the coupled model. For a smaller area of interactive ocean, a shorter lag is expected. Indeed, experiments in which the Indian Ocean or the Atlantic Ocean mixed layer SST is replaced by climatological SST confirm that this is the case. Furthermore, the tropospheric thermal response is smaller when the Indian Ocean is noninteractive than when the Atlantic Ocean is noninteractive, although the area of noninteractive ocean is the same for both runs (by construction), suggesting that the tropical troposphere may have higher sensitivity to the Indian Ocean SST than to the Atlantic Ocean SST (SNM03).

\section{Exploring the coupled nature of the phase lag analytically}

a. A coupled system for the troposphere and mixed layer ocean

In our simulations, the lag of $\left\langle\hat{T}^{\prime}\right\rangle$ with respect to Niño-3.4 SST is a result of the coupled atmosphereocean response to localized SST forcing. Thus, it is tempting to solve the coupled problem in a simple analytical framework to gain insights into the mechanisms for the lagged response.

Following the analytical atmospheric model described in SNM03 and SN03, we add the equation for the mixed layer ocean component and obtain a coupled atmosphere-ocean system as below:

$$
\begin{aligned}
\left(c_{p} P_{T} / g\right)\left[\partial _ { t } \left(\hat{T}^{\prime}\right.\right. & \left.\left.+\hat{q}^{\prime}\right)+\widehat{\mathbf{v} \cdot \hat{\nabla} T^{\prime}}+\widehat{\mathbf{v} \cdot \hat{\nabla} q^{\prime}}+(M \nabla \cdot \mathbf{v})^{\prime}\right] \\
& =R_{s}^{\prime}-R_{t}^{\prime}+E^{\prime}+H^{\prime} \\
C_{M} \partial_{t} T_{M}^{\prime} & =-R_{s}^{\prime}-E^{\prime}-H^{\prime}
\end{aligned}
$$

where tropospheric temperature and moisture are both in kelvins. The carets represents vertical averages within the troposphere and primes denote anomalies from climatological means. The mixed layer ocean SST is $T_{M}$, while $R_{s}$ and $R_{t}$ are radiative fluxes at the surface and TOA, respectively, with a positive sign for upward fluxes. The surface latent and sensible heat fluxes are denoted $E$ and $H$, respectively. The heat capacity of the mixed layer ocean is $C_{M}$.
Applying tropical averages to the above equations and using approximate flux linearization (see appendix A), we can write the rates of change of the tropospheric temperature and mixed layer SST as follows:

$$
\begin{gathered}
C_{a} \partial_{t} \hat{T}^{\prime}+\left[\epsilon_{\mathrm{out}}^{\mathrm{net}}+\left(1-\sigma_{L}\right) \epsilon_{\mathrm{sfc}}^{\mathrm{net}}\right] \hat{T}^{\prime}=\epsilon_{T_{s}}^{\mathrm{net}}\left(\sigma_{M} T_{M}^{\prime}+\sigma_{F} T_{F}^{\prime}\right) \\
C_{M} \partial_{t} T_{M}^{\prime}+\epsilon_{T_{s}}^{\mathrm{net}} T_{M}^{\prime}=\epsilon_{\mathrm{sfc}}^{\mathrm{net}} \hat{T}^{\prime}
\end{gathered}
$$

where

$$
\begin{aligned}
& \epsilon_{\mathrm{out}}^{\mathrm{net}}=\epsilon_{\mathrm{tr}}+\epsilon_{\mathrm{top}}^{\mathrm{net}}, \quad \epsilon_{\mathrm{top}}^{\mathrm{net}}=\epsilon_{T}^{t}+n \epsilon_{q}^{t}, \\
& \epsilon_{\mathrm{sfc}}^{\mathrm{net}}=\epsilon_{H} b+\epsilon_{H} a-\epsilon_{T}^{s}-n \epsilon_{q}^{s}, \\
& \epsilon_{T_{s}}^{\mathrm{net}}=\epsilon_{T_{s}}^{s}+\epsilon_{H} \gamma+\epsilon_{H} .
\end{aligned}
$$

Note that $\hat{T}^{\prime}$ represents tropical averaged tropospheric temperature anomalies, while mixed layer SST $T_{M}$ and specified SST forcing $T_{F}$ are averaged over their respective areas. The area fractions of the mixed layer ocean and the specified SST forcing are $\sigma_{M}$ and $\sigma_{F}$, respectively. The flux exchange coefficient $\epsilon_{\text {out }}^{\text {net }}$ is the coefficient for all energy losses out of the tropical troposphere, combining loss coefficients $\epsilon_{\text {top }}^{\text {net }}$ at TOA and $\epsilon_{\mathrm{tr}}$ to the midlatitudes. The Tropics-midlatitude moist static energy (MSE) flux exchange coefficient $\epsilon_{\text {tr }}$ represents the effect on the total atmospheric column per unit change of tropospheric temperature, similar to radiative fluxes acting on the TOA. The coefficient $\epsilon_{\mathrm{sfc}}^{\text {net }}$ includes the effect of surface radiative fluxes associated with temperature and moisture changes and the counteracting effect of surface air temperature and moisture change on evaporation and sensible heat fluxes. Another coefficient, $\epsilon_{T_{s}}^{\text {net }}$, is associated with the net surface flux changes per unit change of surface temperature. The small TOA flux loss owing to SST $\epsilon_{T_{s}}^{t}$, is omitted. The equivalent atmospheric heat capacity is $C_{a}=$ $\left(c_{p} P_{T} / g\right)(1+n)$, where $n$ is the regression coefficient of $\hat{q}^{\prime}$ on $\hat{T}^{\prime}$.

The values for each flux exchange coefficient are listed in Table 1. They are based on the parameters used in the QTCM or linear fitting to the model simulations. Quantitative aspects of the analytical results may vary as the flux exchange coefficients change, but the general characteristics of the lag and amplitude of the coupled system hold well for reasonable ranges of the coefficients. Note that $\epsilon_{T}^{s}$ and $\epsilon_{q}^{s}$ are negative, corresponding to downward longwave fluxes from the atmosphere to the surface. We ignore cloud-radiative feedback here. Its effect can be considered as a feedback term as in $\mathrm{SN} 02$.

In the rhs of (6.3), contributions from interactive mixed layer SST $\left(T_{M}^{\prime}\right)$ and specified ENSO SST forcing 
TABLE 1. Definitions and values of the flux exchange coefficients for the analytical model $\left(\mathrm{W} \mathrm{m}^{-2} \mathrm{~K}^{-1}\right)$.

\begin{tabular}{llr}
\hline \hline$\epsilon_{T}^{t}$ & TOA longwave flux change per $\hat{T}^{\prime}$ & 3.0 \\
$\epsilon_{q}^{t}$ & TOA longwave flux change per $\hat{q}^{\prime}$ & -1.8 \\
$\epsilon_{T_{s}}^{t}$ & TOA longwave flux change per $T_{s}^{\prime}$ & 0.3 \\
$\epsilon_{T}^{s}$ & Surface longwave flux change per $\hat{T}^{\prime}$ & -3.3 \\
$\epsilon_{q}^{s}$ & Surface longwave flux change per $\hat{q}^{\prime}$ & -5.5 \\
$\epsilon_{T_{s}}^{s}$ & Surface longwave flux change per $T_{s}^{\prime}$ & 6.28 \\
$\epsilon_{H}$ & Coefficient $\left(\rho_{a} C_{H} V_{s}\right)$ for evaporation and sensible & 5.5 \\
& $\quad$ heat fluxes \\
$\epsilon_{t r}$ & Tropics-midlatitude MSE transport regressed \\
& $\quad$ on $\hat{T}^{\prime}$ \\
$\epsilon_{\text {top }}^{\text {net }}$ & Net TOA longwave flux change per $\hat{T}^{\prime}$ and $\hat{q}^{\prime}$ & 5.4 \\
$\epsilon_{\text {out }}^{\text {net }}$ & Net flux loss from the troposphere per $\hat{T}^{\prime}$ & 1.9 \\
$\epsilon_{\text {sfc }}^{\text {net }}$ & Net surface flux change per $\hat{T}^{\prime}$ \\
$\epsilon_{T_{s}}^{\text {net }}$ & Net surface flux change per $T_{s}^{\prime}$ & 7.3 \\
\hline
\end{tabular}

$\left(T_{F}^{\prime}\right)$ both appear. On time scales longer than a day, the averaged surface heat fluxes are zero over land regions owing to small heat capacity. As a result, no contribution from land fraction $\sigma_{L}$ appears in the rhs of (6.3). Similarly, the $\epsilon_{\mathrm{sfc}}^{\text {net }}$ term for the surface flux contributions associated with tropical averaged tropospheric temperature is reduced by the factor $1-\sigma_{L}$ owing to land fraction.

It is clear from (6.3) and (6.4) that the rate of change of tropospheric temperature and mixed layer ocean SST in the coupled system evolves differently from an uncoupled system. For tropospheric temperature alone, its free damping time scale is $C_{a}\left[\epsilon_{\mathrm{out}}^{\mathrm{net}}+\left(1-\sigma_{L}\right) \epsilon_{\mathrm{sfc}}^{\mathrm{net}}\right]^{-1}$, determined by the TOA and surface flux exchanges. For mixed layer SST only, its free damping time scale, $C_{M}\left(\epsilon_{T_{s}}^{\text {net }}\right)^{-1}$, is a function of mixed layer heat capacity and the rate of flux exchange associated with SST variations. The interaction of the troposphere and mixed layer ocean appears in the rhs of (6.3) and (6.4). The mixed layer ocean impacts the tropospheric temperature change through surface fluxes, and the tropospheric temperature affects the mixed layer SST by downward radiative fluxes and surface air temperature and moisture changes, which tend to oppose evaporation and sensible heat flux changes resulting from surface temperature variations. Since the mixed layer ocean has a larger heat capacity than the atmosphere, $T_{M}^{\prime}$ evolves at a slower rate, which slows down the tropospheric temperature response to specified ENSO SST forcing.

\section{b. Implications for coupled evolution and coupled mode decay}

Because the heat capacity of the atmosphere is much smaller than that of the mixed layer ocean, a useful case is to neglect the term $C_{a} \partial_{t} \hat{T}^{\prime}$ and solve for the eigen- value of the linear system. This free-decay rate for the coupled system is

$$
\lambda=\epsilon_{T_{s}}^{\mathrm{net}}\left[\epsilon_{\text {out }}^{\mathrm{net}}+\sigma_{F} \epsilon_{\mathrm{sfc}}^{\mathrm{net}}\right] /\left[C_{M}\left(\epsilon_{\mathrm{out}}^{\mathrm{net}}+\left(1-\sigma_{L}\right) \epsilon_{\mathrm{sfc}}^{\mathrm{net}}\right)\right] .
$$

The overall decay time is determined by a combination of the three exchange coefficients-the mixed layer ocean heat capacity and fractions of land and mixed layer ocean. The term $\sigma_{F} \epsilon_{\text {sfc }}^{\text {net }}$ corresponds to the heat loss from the troposphere to the ocean over the specified SST forcing region. For cases with $\sigma_{F}=0$, for example, a global warming scenario when forcing is given by radiation, the free decay rate of the coupled system becomes $\lambda=\epsilon_{T_{s}}^{\mathrm{net}} \epsilon_{\text {out }}^{\mathrm{net}} /\left[C_{M}\left(\epsilon_{\mathrm{out}}^{\mathrm{net}}+\left(1-\sigma_{L}\right) \epsilon_{\mathrm{sfc}}^{\mathrm{net}}\right)\right]$.

In the QTCM experiments here, land accounts for $26 \%$ of the Tropics $\left(25^{\circ} \mathrm{S}-25^{\circ} \mathrm{N}\right)$, while the mixed layer ocean takes up $61 \%$ of the Tropics. The rest is the specified SST forcing region (13\%). Based on the values of flux exchange coefficients in Table 1, the characteristic time scale of the coupled system is $\tau_{\text {coupled }}=$ $1 / \lambda=3.84 H$, where $H$ is the MLD in meters and $\tau_{\text {coupled }}$ is in days. This yields a decay time scale of 192 days for a MLD of $50 \mathrm{~m}$. If $\sigma_{F}=0, \tau_{\text {coupled }}=5.24 H$, giving a coupled time scale of about 262 days for an MLD of 50 $\mathrm{m}$. For a simpler case, letting $\sigma_{L}=0$ and $\sigma_{F} \rightarrow 0$,

$$
\lambda=\epsilon_{T_{S}}^{\mathrm{net}} \epsilon_{\mathrm{out}}^{\mathrm{net}} /\left[C_{M}\left(\epsilon_{\mathrm{out}}^{\mathrm{net}}+\epsilon_{\mathrm{sfc}}^{\mathrm{net}}\right)\right] .
$$

The corresponding time scale is $\tau_{\text {coupled }}=6.45 \mathrm{H}$. For an MLD of $50 \mathrm{~m}, \tau_{\text {coupled }}$ is 322 days. The coupled time scale is quite sensitive to the area fraction of the mixed layer ocean since this affects the area with heat capacity relative to the area over which energy loss occurs.

The case (6.9) provides a view of the coupled nature of this mode of decay. If either the surface exchange rates $\epsilon_{T_{s}}^{\text {net }}$ and $\epsilon_{\mathrm{sfc}}^{\mathrm{net}}$ are small, or the TOA plus midlatitude loss rate $\epsilon_{\text {out }}^{\text {net }}$ is small, then the slower rate determines the overall decay. In other words, both flux exchange between the mixed layer ocean and atmosphere and the loss from the tropical atmosphere are crucial processes. The coupled time scale is longer than one would expect from linearized SST decay alone, which is given by $C_{M} / \epsilon_{T_{s}}^{\text {net }}$, corresponding to a time scale of 85 days for an MLD of $50 \mathrm{~m}$. The results here are consistent with earlier work by Barsugli and Battisti (1998) in that they also showed that coupling between atmosphere and ocean through surface heat fluxes reduces the internal damping of temperature anomalies.

\section{c. Analytical solutions to the coupled system with sinusoidal SST forcing}

Considering a sinusoidal SST forcing as in (5.1), (6.3) and (6.4) can be solved without neglecting $C_{a}$ to obtain 
the phase lag and amplitude of the tropospheric temperature and mixed layer SST as functions of mixed layer depth, SST forcing period, areal fractional of the mixed layer ocean, and various flux exchange coefficients. The full solution is given in appendix B. Numerical values of the analytical solutions at reasonable mixed layer depths and SST forcing periods are shown in Figs. 13 and 14. Again, we find that the phase lag of tropospheric temperature is not a monotonic function of MLD and maximizes at moderate MLD. It increases as the period of SST forcing increases. The amplitude of temperature response decreases as MLD increases and increases as the forcing period increases. For mixed layer SST, the lag increases monotonically for deeper mixed layer depths. Its amplitude also decreases monotonically with mixed layer depth. Longer period ENSO SST forcing would be associated with longer lag and
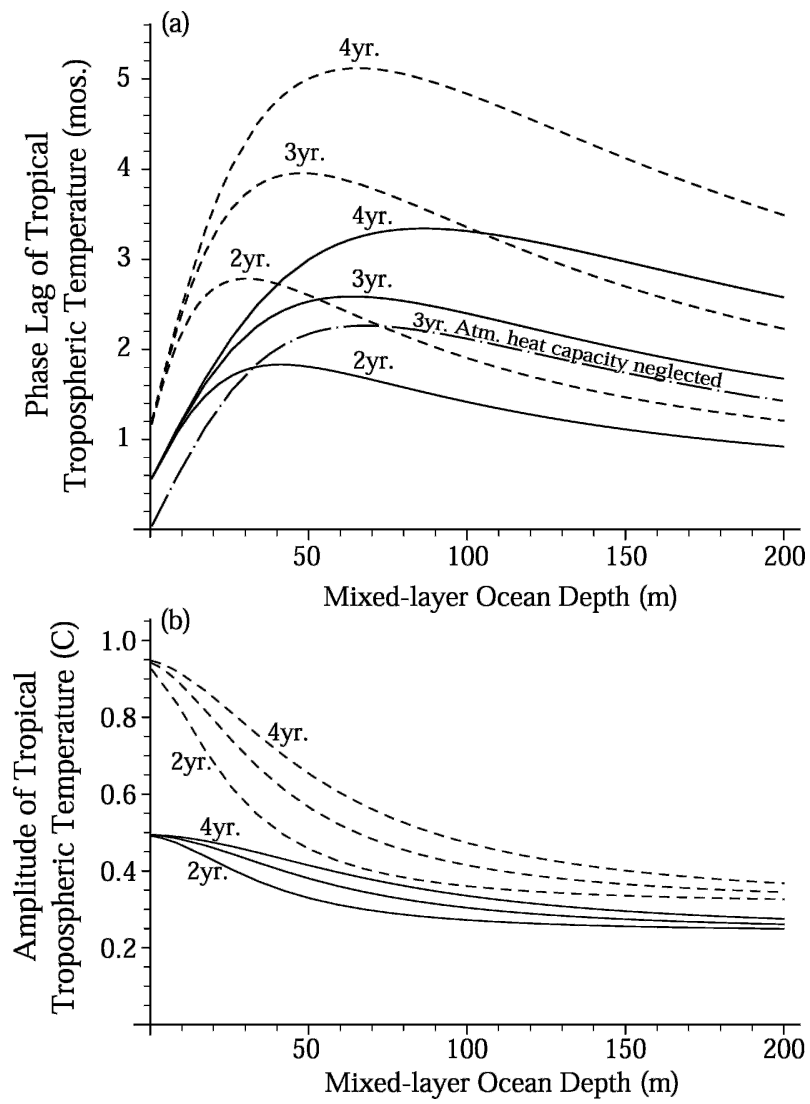

FIG. 13. Analytical model (a) phase lag (months) and (b) amplitude $\left({ }^{\circ} \mathrm{C}\right)$ of tropical averaged tropospheric temperature anomalies as functions of mixed layer depth and SST forcing period. The solid curves correspond to full solutions to (6.4) and (6.5). The dashed curves correspond to results when MSE transports to midlatitudes are ignored. The long-dashed-dotted line corresponds to the lag of tropospheric temperature when atmospheric heat capacity is neglected for a SST forcing period of $3 \mathrm{yr}$.
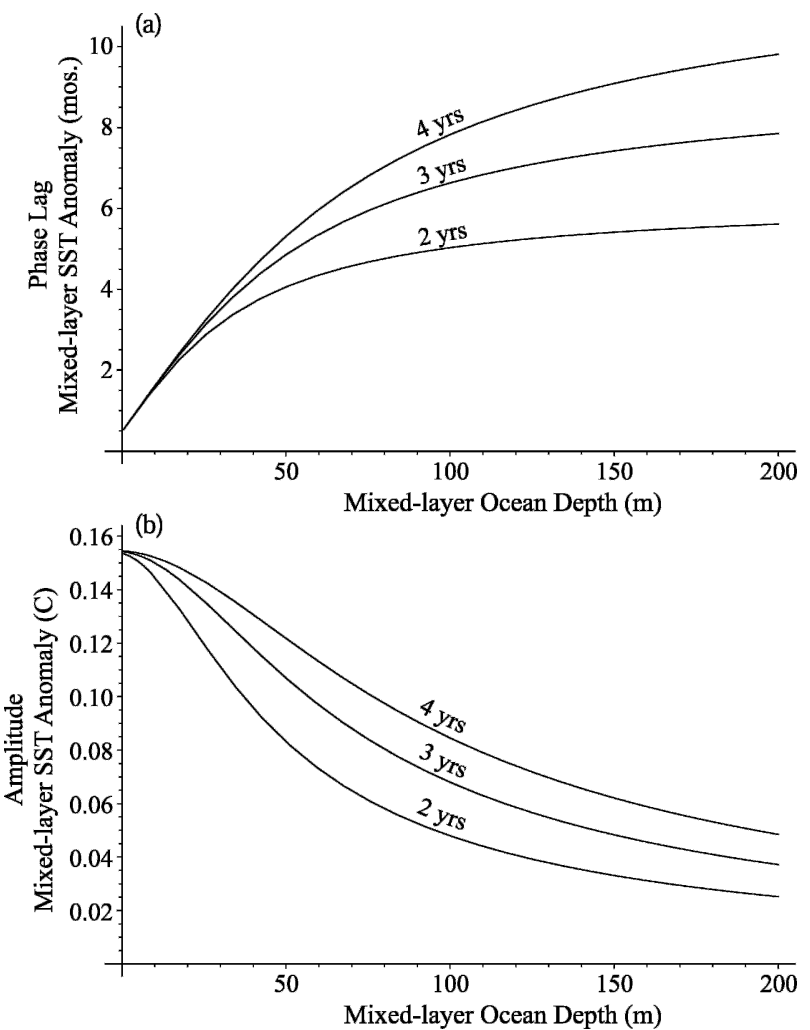

FIG. 14. Analytical model (a) phase lag (months) and (b) amplitude $\left({ }^{\circ} \mathrm{C}\right)$ of mixed layer SST as functions of mixed layer depth and SST forcing period.

stronger amplitude of mixed layer SST. Discussion of scaling the lag curves in Fig. 13a by the period of SST forcing is given in appendix B.

Equations (6.3) and (6.4) give insight into why this system behaves differently as a function of period or mixed layer heat capacity than expected from the usual forced-damped mixed layer ocean. Because the forcing occurs via the atmospheric equation (6.3) and large $C_{M}$ implies small $T_{M}^{\prime}$, the atmosphere approximately responds to $T_{F}^{\prime}$ alone. The lag in $\left\langle\hat{T}^{\prime}\right\rangle$ for large $C_{M}$ (at a given forcing period) is due to the fast atmospheric adjustment and is thus small.

The role of the atmospheric time scale in the phaselag dependence may be seen by comparing the solid curve for a 3-yr period in Fig. 13a (which includes $C_{a}$ ) to the long-dash-dotted curve (which does not). The two curves appear to be shifted by the amount associated with the atmospheric internal heat capacity (corresponding to a time scale of 0.5 months). With $C_{a}=0$, the phase lag goes to zero at both high (not shown) and low values of $C_{M}$. At high $C_{M}$, this occurs because $T_{M}^{\prime} \rightarrow 0$ and there is no ocean effect on the fast atmosphere; at low $C_{M}$ neither fluid has significant heat ca- 
pacity. When $C_{a} \neq 0$, the atmospheric time scale limits how small the phase lag can get as $C_{M}$ varies.

Another useful case to consider for (6.3)-(6.4) is a switch-on problem where $T_{F}^{\prime}$ is increased suddenly and then held constant. Because atmosphere and mixedlayer ocean time scales are very different, initially $T_{M}^{\prime}$ remains small, while $\hat{T}^{\prime}$ increases rapidly to balance with $T_{F}^{\prime}$. Then as $T_{M}^{\prime}$ equilibrates on time scale $\tau_{\text {coupled }}$, $\hat{T}^{\prime}$ can increase further in balance with $T_{F}^{\prime}$ plus the current value of $T_{M}^{\prime}$ in (6.3). Thus $\hat{T}^{\prime}$ has two stages of warming: one fast, but limited by energy loss to $T_{M}^{\prime}$, and one slow, on a slaved coupled time scale, limited by energy loss to space and midlatitudes.

Comparing Figs. 13 and 14 to Figs. 11 and 12, the analytical model results of phase lag and amplitude are consistent with those from the numerical simulations, although exact values differ. This may be due to our simplified flux linearization, averaging over atmospheric wave dynamics, and neglect of cloud-radiative feedback, etc.

\section{d. Precipitation}

It is possible to solve the tropical averaged precipitation anomalies in the coupled system using (6.3) and a moisture equation as in SN03 [their Eq. (3.2)]. The resulting tropical mean precipitation is

$$
\begin{aligned}
\left\langle P^{\prime}\right\rangle= & C_{a}(1+n)^{-1} \partial_{t} \hat{T}^{\prime}+\left[\epsilon_{\mathrm{out}}^{T \text { net }}+\left(1-\sigma_{L}\right) \epsilon_{\mathrm{sfc}}^{\text {Tnet }}\right] \hat{T}^{\prime} \\
& -\epsilon_{T_{s}}^{T \text { net }}\left(\sigma_{M} T_{M}^{\prime}+\sigma_{F} T_{F}^{\prime}\right),
\end{aligned}
$$

where the flux exchanges coefficients $\epsilon_{\text {out }}^{T \text { net }}, \epsilon_{\text {sfc }}^{T \text { net }}$, and $\epsilon_{T_{s}}^{T \text { net }}$ are similar to their counterparts in (6.3) except they that do not include the moisture transport between the Tropics and midlatitudes and terms associated with evaporation:

$$
\begin{aligned}
& \epsilon_{\mathrm{out}}^{T \text { net }}=\epsilon_{\mathrm{tr}}^{T}+\epsilon_{T}^{t}+n \epsilon_{q}^{t}, \\
& \epsilon_{\mathrm{sfc}}^{T \text { net }}=\epsilon_{H} a-\epsilon_{T}^{s}-n \epsilon_{q}^{s}, \\
& \epsilon_{T_{s}}^{T \text { net }}=\epsilon_{T_{s}}^{s}+\epsilon_{H} .
\end{aligned}
$$

The coefficient $\epsilon_{t r}^{T}$ is the regression coefficient of Tropics-midlatitude dry static energy transport onto $\left\langle\hat{T}^{\prime}\right\rangle$,

$$
\left(c_{p} P_{T} / g\right)\left\langle\mathbf{v} \cdot \hat{\nabla} T^{\prime}+\left(M_{s} \nabla \cdot \mathbf{v}\right)^{\prime}\right\rangle \approx \epsilon_{t r}^{T}\left\langle\hat{T}^{\prime}\right\rangle,
$$

where $M_{s}$ is the "gross dry stratification" (Neelin and Zeng 2000). The value of $\epsilon_{\mathrm{tr}}^{T}$ is usually larger than $\epsilon_{\mathrm{tr}}$ because the averaged dry static energy transport tends to cancel with moisture transport.

Substituting for $\left\langle\hat{T}^{\prime}\right\rangle$ using (6.3), and assuming a sinusoidal SST forcing of frequency $\omega$, we have

$$
\begin{aligned}
\left\langle P^{\prime}\right\rangle= & \left(\frac{\left[\frac{C_{a}}{(1+n)} i \omega+\epsilon_{\mathrm{out}}^{T \mathrm{net}}+\left(1-\sigma_{L}\right) \epsilon_{\mathrm{sfc}}^{T \mathrm{net}}\right.}{\left[C_{a} i \omega+\epsilon_{\mathrm{out}}^{\mathrm{net}}+\left(1-\sigma_{L}\right) \epsilon_{\mathrm{sfc}}^{\mathrm{net}}\right] \epsilon_{T_{s}}^{\mathrm{net}}-\epsilon_{T_{s}}^{T \text { net }}}\right) \\
& \times\left(\sigma_{M} T_{M}^{\prime}+\sigma_{F} T_{F}^{\prime}\right) .
\end{aligned}
$$

In the above expression, the tropical mean precipitation anomalies depend on a weighted combination of SST anomalies in forcing and mixed layer regions. The proportionality factor results from the canceling effects of various flux coefficients. Depending on the exact values of these coefficients, the real part of this factor can be positive or negative and tends to be small. With the values of flux exchange coefficients, as in Table 1, the tropical mean precipitation has a lag relative to the specified SST forcing that lies between the lag of tropospheric temperature and the lag of mixed layer SST. The lag of precipitation anomalies does not control the lag for tropospheric temperature. For example, keeping the same MSE transport rate $\left(\epsilon_{\mathrm{tr}}\right)$ and varying the moisture transport, changes in $\epsilon_{t r}^{T}$ would give different precipitation lag but have no effect in tropospheric temperature lag. This, again, supports the view that tropical mean precipitation is not a driving force for tropospheric temperature change. It is a consequence of moist convective adjustment to interannual SST forcing and midlatitude transports.

\section{Role of midlatitude transports}

The moist static energy transport from the Tropics is not a dominant term in budgets for seasonal tropospheric temperature anomaly (SNM03), but such transport has a nonnegligible role in determining the lag of tropospheric temperature relative to SST. The linear regression of tropical-averaged moist static energy transport anomalies onto the tropical-averaged tropospheric temperature anomalies yields a coefficient of $5.4 \mathrm{~W} \mathrm{~m}^{-2} \mathrm{~K}^{-1}$, comparable to the coefficient for the TOA radiative flux associated with tropospheric temperature changes. The moist static energy transport anomalies act as a damping to the tropospheric temperature change. The equivalent damping time scale is about 10 days. Without moist static energy transport, the analytical results yield a much longer phase lag and a larger amplitude of tropospheric temperature response. For example, the phase lag and amplitude of $\left\langle\hat{T}^{\prime}\right\rangle$ increases by $50 \%$ when the MSE transport is ignored for a SST forcing period of $3 \mathrm{yr}$ shown in Fig. 13a (dashed line). A QTCM experiment (run ADVCLIM) is conducted in which the anomalies of MSE transport are reduced by replacing advection terms $\mathbf{v} \cdot \hat{\nabla} T^{\prime}$ and $\mathbf{v} \cdot \hat{\nabla} q^{\prime}$ by their climatological values. The resulting 


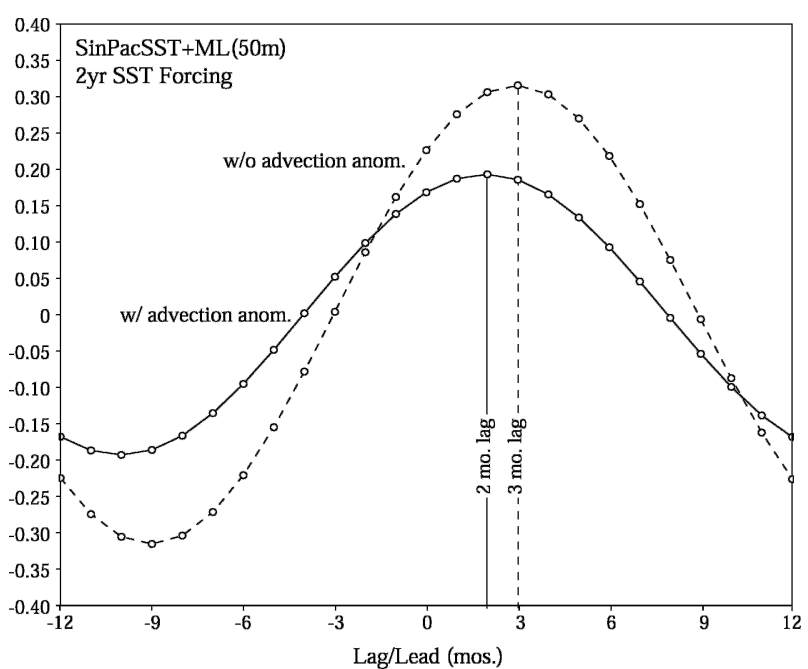

FIG. 15. Lead/lag regression coefficients of tropical tropospheric temperature anomalies onto the Niño-3.4 SST index for the QTCM run ObsPac+ML $($ MLD $=50 \mathrm{~m})$ with and without advection anomalies. Corresponding lags for maximum regression values are marked by dashed lines.

lead/lag regression of $\left\langle\hat{T}^{\prime}\right\rangle$ relative to Niño-3.4 SST forcing is shown in Fig. 15. Compared to the corresponding control run with a lag of 2 months, the temperature lag increases to 3 months and the anomaly amplitude is increased by $60 \%$. Thus, the MSE transport between the Tropics and midlatitudes is an important process limiting the thermal response of the troposphere to the ENSO SST forcing.

\section{Summary and discussion}

Tropical averaged tropospheric temperature variations lag ENSO SST in the eastern Pacific by a few months. SST in other basins also exhibits a lag relationship with the eastern Pacific SST. While SST variability in other basins is not solely controlled by ENSO, it is useful to analyze the relationships in a coupled atmosphere-mixed layer ocean system, where the ENSO region SST is specified as a forcing. Although the tropospheric temperature lag exists even when no atmosphere-ocean coupling is allowed (i.e., in run ObsPac), coupling with an ocean mixed layer slows the tropospheric response to a significant extent. This is consistent with the numerical experiments presented in KH03. The pattern of tropospheric temperature warming resembles that expected from wave dynamics, but warming outside the ENSO region increases on a time scale longer than that of the atmospheric wave adjustment process. A number of numerical experiments and a simple analytical model are used to examine the mechanism and sensitivity of the phase lag and amplitude of the tropospheric temperature response to ENSO SST forcing. The QTCM and approximate analytical results are roughly comparable to that observed, using peak response as a measure of lag.

It is found that tropospheric temperature lag is not a monotonic function of mixed layer depth. It maximizes at moderate mixed layer depths, about $25-100 \mathrm{~m}$. In the limit of large or small mixed layer depth, the phase lag asymptotes to values given by free atmospheric time scales. In QTCM results, the lag is 1.5 months for small MLD. With observed ENSO SST forcing, the QTCMsimulated tropospheric temperature phase lag varies with MLD rather insensitively, ranging from 1.5 months for very small MLD to 2.5 months for the $25-100 \mathrm{~m}$ of MLD range, and dropping again for larger MLD (Fig. 7). The amplitude of $\left\langle\hat{T}^{\prime}\right\rangle$ decreases as MLD increases. For the mixed layer SST, increasing MLD yields a monotonically longer lag and much smaller amplitude. The small mixed layer SST response adds little to the atmospheric warming, which is why the tropospheric temperature lag becomes small when MLD is sufficiently large. The phase lag and amplitude of the tropospheric temperature response appear not to be sensitive to the SST seasonal cycle. Area fractions of interactive ocean and land impact the response time scale significantly. In a case when anomalies of temperature and moisture advection from the Tropics to midlatitudes are suppressed in the model, the phase lag increases substantially. In experiments with sinusoidal SST forcing, the phase lag and amplitude of $\left\langle\hat{T}^{\prime}\right\rangle$ increase significantly with forcing period.

In these results, tropical mean rainfall anomalies have a different phase relationship to ENSO SST than to tropospheric temperature anomalies. The tropical mean precipitation anomalies are residuals of large compensation between positive anomalies within the ENSO forcing region and negative anomalies outside, while both quantities exhibit little lag behavior with respect to ENSO SST forcing. These results appear consistent with the argument of SN03 that the tropical mean precipitation anomaly is not a driver for the tropospheric temperature change, but a by-product of the atmosphere and ocean coupled response to interannual SST forcing. We also find that the lag of tropospheric temperature is not sensitive to shifts in the phase of peak SST warming relative to the seasonal cycle. Our results thus differ from postulates by $\mathrm{KH} 03$ regarding the role of precipitation and of the seasonal cycle of SST, although we agree with $\mathrm{KH} 03$ that the interaction of the atmosphere with the Indian and Atlantic Oceans is an important part of the coupled response. However, interaction with other basins appears not to be the lead- 


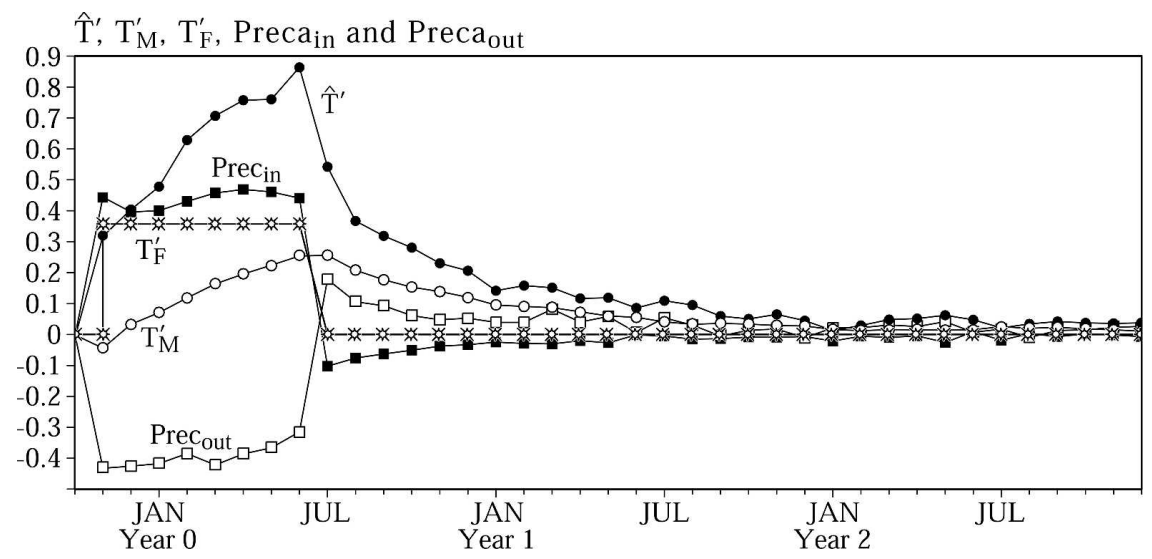

FIG. 16. Evolution of tropical averaged tropospheric temperature $\left(\hat{T}^{\prime}\right)$, mixed layer SST $\left(T_{M}^{\prime}\right)$, and precipitation anomalies inside $\left(\operatorname{Prec}_{\text {in }}\right)$ and outside $\left(\mathrm{Prec}_{\mathrm{out}}\right)$ the ENSO SST forcing region, along with the specified SST forcing $\left(T_{F}^{\prime}\right)$ for a QTCM simulation with an ENSO SST anomaly switched on for 8 months and then switched off.

ing cause for the asymmetric tropospheric temperature response before and after observed peak warming. In our runs with observed SST anomalies specified, the asymmetry is quite evident, but it vanishes when an idealized sinusoidal SST forcing is used. Thus, it appears to result from the asymmetry in ENSO onset versus decay in SST.

An interesting feature emerges when we examine the sensitivity of the precipitation response to ENSO SST forcing for different mixed layer depths. For deeper mixed layer depth, both precipitation anomalies inside and outside the ENSO forcing region exhibit greater amplitude (Fig. 9). The response outside the ENSO region is consistent with Chiang and Sobel (2002) in that a larger mixed layer heat capacity permits greater disequilibrium between atmosphere and ocean, with larger surface fluxes giving rise to larger precipitation anomalies. In both studies, convection is important in connecting the lower boundary and troposphere, but the amount of diabatic heating anomalies (precipitation anomalies) depends on interaction with the ocean. In results here, tropospheric temperature is interactive and feeds back on the precipitation both inside and outside the ENSO forcing region.

Using a simply analytical model framework, it is clear that the exchange of fluxes at the atmosphere-ocean interface and the atmospheric energy loss to space and to the midlatitudes greatly impact the time scales at which the atmosphere and ocean respond to external forcing. As a result, the coupled time scale involves the combined effect of radiative fluxes at the top of the atmosphere and at the surface, surface heat fluxes, and Tropics-midlatitude moist static energy transports, compounded with mixed layer heat capacity.
The slowing of the decay rate of the tropospheric temperature by the ocean is illustrated in a simple experiment, which-interpreted using analytical model (6.3) and (6.4) results-summarizes the underlying physics well enough that we use it as a conclusion. In this experiment, we impose a constant positive SST anomaly of $2^{\circ}$ inside the composite ENSO SST region for the first 8 months, then turn it off afterward. The rest of the ocean is a 50-m mixed layer. The response time series of tropical-averaged tropospheric temperature and mixed layer SST are plotted in Fig. 16. A component of $\left\langle\hat{T}^{\prime}\right\rangle$ responds fairly quickly, within in a month or two. This time scale is associated with fast surface flux adjustment and wave dynamics for tropospheric temperature only. As other ocean regions warm owing to teleconnected surface flux change and cloudradiative feedback, the $\left\langle\hat{T}^{\prime}\right\rangle$ and mixed layer SST have a slower, covarying signal. The warming of the coupled system peaks near the end of the positive ENSO forcing and stays positive for a substantial period after the SST forcing is turned off. After an initial fast decay of part of the atmospheric signal, there is a roughly exponential joint decay of $\left\langle\hat{T}^{\prime}\right\rangle$ and $T_{M}^{\prime}$ with a time scale of about half a year. On the other hand, the precipitation has characteristics different from $\left\langle\hat{T}^{\prime}\right\rangle$ and $T_{M}^{\prime}$. It has a sudden response in the beginning to the SST forcing, then evolves more or less along with the combined mixed layer ocean and tropospheric temperature changes.

The exponential growth/decay of the mixed layer SST and tropospheric temperature that appears in both the spinup and decay phases is consistent with a coupled tropospheric temperature and SST mode found in the analytical model. The characteristic time scale $\tau_{\text {coupled }}$ of this coupled mode depends on SST- 
troposphere flux exchange coefficients and coefficients for the TOA and midlatitude energy loss, as noted in (6.8). Both the flux exchange between the mixed layer ocean and atmosphere and the loss from the tropical atmosphere to space and to midlatitudes are important processes determining the coupled mode contribution to the lag of tropospheric temperature. This coupled mode contribution, due to interaction with SST outside the ENSO region, substantially increases the amplitude and lag of the tropospheric temperature response to ENSO.

Acknowledgments. This work was supported under National Science Foundation Grant ATM-0082529, National Oceanographic and Atmospheric Administration Grants NA16-GP2003, NA0OAR4310013, and NA05OAR4311134. The authors thank A. Kumar and M. P. Hoerling for sharing their prepublished manuscript. Discussions with F.-F. Jin and A. H. Sobel are appreciated.

\section{APPENDIX A}

\section{Approximate Linearization to Flux Exchange Terms}

To solve (6.1) and (6.2) analytically, we need to linearize the flux exchange terms. Because tropical averaged moisture anomalies are approximately linear with tropospheric temperature anomalies, we use $\left\langle\hat{q}^{\prime}\right\rangle \approx n$ $\left\langle\hat{T}^{\prime}\right\rangle$ with $n \approx 0.6$. The angle brackets denote tropical averages. The tropical-averaged perturbation surface fluxes and radiative fluxes can be approximately linearized as functions of tropospheric temperature, moisture, and SST using empirical flux exchange coefficients as in SNM03 and SN03. The tropical-midlatitude heat and moisture transport terms in (6.1) can also be linearly regressed on temperature anomalies with a parameter $\epsilon_{\mathrm{tr}}$. Thus,

$$
\begin{aligned}
\left(c_{p} P_{T} / g\right)\left\langle\widehat{\mathbf{v} \cdot \hat{\nabla} T^{\prime}}\right. & \left.+\widehat{\mathbf{v} \cdot \hat{\nabla} q^{\prime}}+(M \nabla \cdot \mathbf{v})^{\prime}\right\rangle \approx \epsilon_{t r}\left\langle\hat{T}^{\prime}\right\rangle, \\
R_{s, t}^{\prime} & \approx \epsilon_{T}^{s, t} \hat{T}^{\prime}+\epsilon_{q}^{s, t} \hat{q}^{\prime}+\epsilon_{T_{s}}^{s, t} T_{s}^{\prime}, \\
E^{\prime} & \approx \rho_{a} C_{H} \mathrm{~V}_{s}\left[q_{s a t}^{\prime}\left(T_{s}\right)-q_{a s}^{\prime}\right] \\
& \approx \epsilon_{H} \gamma T_{s}^{\prime}-\epsilon_{H} b \hat{T}^{\prime} \\
H^{\prime} & \approx \rho_{a} C_{H} \mathrm{~V}_{s}\left[T_{s}^{\prime}-T_{a s}^{\prime}\right] \\
& \approx \epsilon_{H} T_{s}^{\prime}-\epsilon_{H} a \hat{T}^{\prime} .
\end{aligned}
$$

We use $\epsilon_{H}=\rho_{a} C_{H} V_{s}$ for the surface flux feedback coefficient. The factors $a$ and $b$ represent the ratio of surface air temperature and moisture to tropospheric mean temperature, respectively, with $a \approx 0.7$ and $b \approx 2.0$. The parameter $\gamma=\left(d q_{\mathrm{sat}} / d T\right)_{T s}$ and is about
$3 \mathrm{~K} \mathrm{~K}^{-1}$ for the normal range of SST variations. Note that we ignore the evaporation (and sensible heat flux) changes due to surface wind for simplicity, although this term may not be negligible for many cases of ENSO teleconnection (SN02; Neelin and Su 2005).

In (A.2), (A.3), and (A.4), tropical averages are not given explicitly because we use the same flux exchange coefficients for all tropical regions. The linearization applies locally as well. For compactness, variables in later sections represent tropical averages, except for mixed layer SST $T_{M}$ and specified SST forcing $T_{F}$, which are averaged over their respective areas.

\section{APPENDIX B}

\section{Solutions to the Analytical Model}

The dynamics of the coupled atmosphere mixed layer ocean in response to a sinusoidal SST forcing can be described by the following equations:

$$
\begin{aligned}
\partial_{t} T^{\prime}+m_{11} T^{\prime}+m_{12} T_{M}^{\prime} & =\sigma_{F} \epsilon_{T_{s}}^{\text {net }} T_{s 0} \sin (\omega t) / C_{a}, \\
\partial_{t} T_{M}^{\prime}+m_{21} T^{\prime}+m_{22} T_{M}^{\prime} & =0,
\end{aligned}
$$

where the amplitude of the SST forcing is $T_{s 0}$ and $\omega$ is the forcing frequency. For simplicity, we use $f_{0}=$ $\sigma_{F} \epsilon_{T_{s}}^{\text {net }} T_{s 0} / C_{a}$ in derivations below. The coefficients $m_{\mathrm{ij}}$ $(i=1,2 ; j=1,2)$ are combinations of flux exchange coefficients and heat capacity of the atmosphere or the mixed layer ocean,

$$
\begin{aligned}
& m_{11}=\left[\epsilon_{\mathrm{out}}^{\mathrm{net}}+\left(1-\sigma_{L}\right) \epsilon_{\mathrm{sfc}}^{\mathrm{net}}\right] / C_{a} \\
& m_{12}=-\sigma_{M} \epsilon_{T_{s}}^{\mathrm{net}} / C_{a} \\
& m_{21}=-\epsilon_{\mathrm{sfc}}^{\mathrm{net}} / C_{M} \\
& m_{22}=\epsilon_{T_{S}}^{\mathrm{net}} / C_{M} .
\end{aligned}
$$

It is straightforward to solve the set of linear equations (6.3) and (6.4) in response to a sinusoidal SST forcing:

$$
\begin{aligned}
\hat{T}^{\prime}(t)= & \hat{T}^{\prime}(\omega) \sin \left(\omega t-\Delta \phi_{T}\right)+C_{T}^{+} \exp \left(-\gamma_{+} t\right) \\
& +C_{T}^{-} \exp \left(-\gamma_{-} t\right) \\
T_{M}^{\prime}(t)= & T_{M}^{\prime}(\omega) \sin \left(\omega t-\Delta \phi_{T_{M}}\right)+C_{T_{M}}^{+} \exp \left(-\gamma_{+} t\right) \\
& +C_{T_{M}}^{-} \exp \left(-\gamma_{-} t\right),
\end{aligned}
$$

where $\gamma_{ \pm}$are the damping rates in the coupled system, which are the eigenvalues of matrix $m_{\mathrm{ij}}(i=1,2$; $j=1,2)$, 


$$
\gamma_{ \pm}=\frac{1}{2}\left[\left(m_{11}+m_{22}\right) \pm \sqrt{\left(m_{11}-m_{22}\right)^{2}+4 m_{12} m_{21}}\right] .
$$

The amplitudes $C_{\hat{T}}^{ \pm}$and $C_{T_{M}}^{ \pm}$can be found according to the initial conditions. The first terms in Eq. (B.7) are the inhomogeneous terms, which is the long-term solution, while the remaining two terms are the homogeneous solutions that decay away as $t$ becomes much larger than the damping scales. The lag phases and amplitudes for the inhomogeneous terms are expressed as

$$
\begin{aligned}
\hat{T}(\omega) e^{-i \Delta \phi \hat{T}} & =\frac{f_{0}\left(i \omega+m_{22}\right)}{\left(i \omega+m_{11}\right)\left(i \omega+m_{22}\right)-m_{12} m_{21}}, \\
T_{M}(\omega) e^{-i \Delta \phi_{T_{M}}} & =-\frac{f_{0} m_{21}}{\left(i \omega+m_{11}\right)\left(i \omega+m_{22}\right)-m_{12} m_{21}} .
\end{aligned}
$$

Now let us study the lag as a function of the frequency of the external forcing $\omega$. Note that the lag time is simply the phase divided by $\omega$, using Eqs. (B.9) and (B.10),

$$
\begin{aligned}
\operatorname{lag}_{\hat{T}}(\omega) & \equiv \Delta \phi_{\hat{T}} \\
& =\frac{1}{\omega} \tan ^{-1}\left[\frac{\omega\left(\omega^{2}+m_{22}^{2}+m_{12} m_{21}\right)}{m_{11} \omega^{2}+m_{22}\left(m_{11} m_{22}-m_{12} m_{21}\right)}\right], \\
\operatorname{lag}_{T_{M}}(\omega) & \equiv \frac{\Delta \phi_{T_{M}}}{\omega}=\frac{1}{\omega} \cot ^{-1}\left[\frac{m_{11} m_{22}-m_{12} m_{21}-\omega^{2}}{\omega\left(m_{11}+m_{22}\right)}\right] .
\end{aligned}
$$

When we ignore the rate of change of tropospheric temperature owing to the small heat capacity of air, the decay rate of the coupled system is

$$
\lambda=\left(m_{11} m_{22}-m_{12} m_{21}\right) / m_{11},
$$

which yields (6.8). The solutions for $\left\langle\hat{T}^{\prime}\right\rangle$ and $T_{M}$ are

$$
\begin{aligned}
\hat{T}^{\prime} & =\frac{\left(i \omega+m_{22}\right)}{m_{11}(i \omega+\lambda)} f_{0} \\
T_{M}^{\prime} & =\frac{-m_{21}}{m_{11}(i \omega+\lambda)} f_{0} .
\end{aligned}
$$

Thus the phase lag of $\left\langle\hat{T}^{\prime}\right\rangle$ is

$$
\operatorname{lag}_{\hat{T}}=\omega^{-1}\left(\tan ^{-1} m_{22} / \omega-\tan ^{-1} \lambda / \omega\right) .
$$

Note that since there is a single time derivative term in this approximation, one can rescale the equation as follows: $\operatorname{lag}_{\hat{T}}^{*}=\omega \operatorname{lag}_{\hat{T}}, m_{22}^{*}=C_{M} m_{22}, \lambda^{*}=C_{M} \lambda, \omega^{*}=$ $C_{M} \omega$ and, thus, obtain a function independent of $C_{M}$,

$$
\operatorname{lag} \Delta \phi_{\hat{T}}^{*}=\left(\tan ^{-1} m_{22}^{*} / \omega^{*}-\tan ^{-1} \lambda^{*} / \omega^{*}\right) .
$$

Hence, rescaling phase lag and $C_{M}$ by the forcing period in Fig. 13a would yield one curve if the effects of the atmospheric time scale associated with $C_{a}$ were negligible.

\section{REFERENCES}

Alexander, M. A., I. Blade, M. Newman, J. R. Lanzante, N.-C. Lau, and J. D. Scott, 2002: The atmospheric bridge: The influence of ENSO teleconnections on air-sea interaction over the global oceans. J. Climate, 15, 2205-2231.

Angell, J. K., 1981: Comparison of variations in atmospheric quantities with sea surface temperature variations in the equatorial eastern Pacific. Mon. Wea. Rev., 109, 230-243.

Bantzer, C. H., and J. M. Wallace, 1996: Intraseasonal variability in tropical mean temperature and precipitation and their relation to the tropical 40-50 day oscillation. J. Atmos. Sci., 53, 3032-3045.

Barsugli, J. J., and D. S. Battisti, 1998: The basic effects of atmosphere-ocean thermal coupling on midlatitude variability. $J$. Atmos. Sci., 55, 477-493.

Chiang, J., and A. H. Sobel, 2002: Tropical tropospheric temperature variations caused by ENSO and their influence on the remote tropical climate. J. Climate, 15, 2616-2631.

Enfield, D. B., and D. A. Mayer, 1997: Tropical Atlantic sea surface temperature variability and its relation to El NiñoSouthern Oscillation. J. Geophys. Res., 102, 929-945.

Hansen, J., I. Fung, A. Lacis, D. Rind, S. Lenedeff, R. Ruedy, and G. Russell, 1988: Global climate changes as forecast by Goddard Institute for Space Studies three-dimensional model. $J$. Geophys. Res., 93, 9341-9364.

—, R. Ruedy, A. Lacis, G. Russell, M. Sato, J. Lerner, D. Rind, and P. Stone, 1997: Wonderland climate model. J. Geophys. Res., 102, 6823-6830.

Heckley, W. A., and A. E. Gill, 1984: Some simple analytical solutions to the problem of forced equatorial long waves. Quart. J. Roy. Meteor. Soc., 110, 203-217.

Jin, J., and B. Hoskins, 1995: The direct response to tropical heating in a baroclinic atmosphere. J. Atmos. Sci., 52, 307-319.

Kalnay, E., and Coauthors, 1996: The NCEP/NCAR 40-Year Reanalysis Project. Bull. Amer. Meteor. Soc., 77, 437-471.

Klein, S. A., B. J. Soden, and N.-C. Lau, 1999: Remote sea surface temperature variations during ENSO: Evidence for a tropical atmospheric bridge. J. Climate, 12, 917-932.

Kumar, A., and M. P. Hoerling, 2003: The nature and causes for the delayed atmospheric response to El Niño. J. Climate, 16, 1391-1403.

Latif, M., and T. P. Barnett, 1995: Interactions of the tropical oceans. J. Climate, 8, 952-968.

Lau, N.-C., and M. J. Nath, 1996: The role of the "atmospheric bridge" in linking tropical Pacific ENSO events to extratropical SST anomalies. J. Climate, 9, 2036-2057.

Neelin, J. D., and N. Zeng, 2000: A quasi-equilibrium tropical circulation model-Formulation. J. Atmos. Sci., 57, 17411766.

— , and H. Su, 2005: Moist teleconnection mechanisms for the tropical South American and Atlantic sector. J. Climate, 18, 3928-3950

— , F.-F. Jin, and H.-H. Syu, 2000: Variations in ENSO phase locking. J. Climate, 13, 2570-2590. 
, C. Chou, and H. Su, 2003: Tropical drought regions in global warming and El Niño teleconnections. Geophys. Res. Lett., 30, 2275, doi:10.1029/2003GL018625.

Newell, R. E., and B. C. Weare, 1976: Ocean temperatures and large scale atmospheric variations. Nature, 262, 40-41.

Pan, Y. H., and A. H. Oort, 1983: Global climate variations connected with sea surface temperature anomalies in the eastern equatorial Pacific Ocean for the 1958-73 period. Mon. Wea. Rev., 111, 1244-1258.

Rasmusson, E. M., and T. H. Carpenter, 1982: Variations in tropical sea surface temperature and surface wind fields associated with the Southern Oscillation/El Niño. Mon. Wea. Rev., 110, 354-384.

Reid, G. C., K. S. Gage, and J. R. McAfee, 1989: The thermal response of the tropical atmosphere to variations in equatorial Pacific sea-surface temperature. J. Geophys. Res., 94, 14 705-14 716.

Reynolds, R. W., and T. M. Smith, 1994: Improved global sea surface temperature analyses using optimum interpolation. $J$. Climate, 7, 929-948.

Sobel, A. H., and H. Gildor, 2003: A simple time-dependent model of SST hot spots. J. Climate, 16, 3978-3992.

$\mathrm{Su}, \mathrm{H}$., and J. D. Neelin, 2002: Teleconnection mechanisms for tropical Pacific descent anomalies during El Niño. J. Atmos. Sci., 59, 2682-2700.

, and,- 2003 : The scatter in tropical average precipitation anomalies. J. Climate, 16, 3966-3977.
,-- , and C. Chou, 2001: Tropical teleconnection and local response to SST anomalies during the 1997-1998 El Niño. $J$. Geophys. Res., 106, 20 025-20043.

- - - and J. E. Meyerson, 2003: Sensitivity of tropical tropospheric temperature to sea surface temperature forcing. $J$. Climate, 16, 1283-1301.

Tourre, Y. M., and W. B. White, 1995: ENSO signals in global upper-ocean temperature. J. Phys. Oceanogr., 25, 1317-1332.

Watterson, I. G., 2002: The sensitivity of subannual and intraseasonal tropical variability to model ocean mixed-layer depth. J. Geophys. Res., 107, 4020, doi:10.1029/2001JD000671.

Webster, P. J., A. M. Moore, J. P. Loschnigg, and R. R. Leben, 1999: Coupled ocean-atmosphere dynamics in the Indian Ocean during 1997-98. Nature, 401, 356-360.

Yu, J., C. R. Mechoso, J. C. McWilliams, and A. Arakawa, 2002: Impacts of the Indian Ocean on the ENSO cycle. Geophys. Res. Lett., 29, 1204, doi:10.1029/2001GL014098.

Yu, L., and M. M. Rienecker, 2000: Indian Ocean warming of 1997-1998. J. Geophys. Res., 105, $16923-16939$.

Yulaeva, E., and J. M. Wallace, 1994: The signature of ENSO in global temperature and precipitation fields derived from the microwave sounding unit. J. Climate, 7, 1719-1736.

Zeng, N., J. D. Neelin, and C. Chou, 2000: A quasi-equilibrium tropical circulation model-Implementation and simulation. J. Atmos. Sci., 57, 1767-1796. 